\title{
DEFLAGRATION TO DETONATION TRANSITION IN THERMONUCLEAR SUPERNOVAE
}

\author{
A.M. Khokhlov \\ Laboratory for Computational Physics and Fluid Dynamics, \\ Code 6404, Naval Research Laboratory, \\ Washington, DC 20375 \\ E.S. Oran \\ Laboratory for Computational Physics and Fluid Dynamics, \\ Code 6404, Naval Research Laboratory, \\ Washington, DC 20375 \\ J.C. Wheeler \\ Astronomy Department, The University of Texas \\ Austin, TX 78712-1083
}

Received _ ; accepted

Submitted to the Astrophysical Journal 


\begin{abstract}
We derive the criteria for deflagration to detonation transition (DDT) in a Type Ia supernova. The theory is based on the two major assumptions: (i) detonation is triggered via the Zeldovich gradient mechanism inside a region of mixed fuel and products, (ii) the mixed region is produced by a turbulent mixing of fuel and products either inside an active deflagration front or during the global expansion and subsequent contraction of an exploding white dwarf.

We determine the critical size of the mixed region required to initiate a detonation in a degenerate carbon-oxygen mixture. This critical length is much larger than the width of the reaction front of a Chapman-Jouguet detonation. However, at densities greater than $\simeq 5 \times 10^{6} \mathrm{~g} \mathrm{~cm}^{-3}$, it is much smaller than the size of a white dwarf. We derive the critical turbulent intensity required to create the mixed region inside an active deflagration front in which a detonation can form. We conclude that the density $\rho_{\text {tr }}$ at which a detonation can form in a carbon-oxygen white dwarf is low, less than $2-5 \times 10^{7} \mathrm{~g} \mathrm{~cm}^{-3}$, but greater than $5 \times 10^{6} \mathrm{~g} \mathrm{~cm}^{-3}$.
\end{abstract}

Subject headings: detonation, deflagration, supernovae, combustion 


\section{Introduction}

The standard model for Type Ia supernovae (SN Ia) is an explosion by a process of thermonuclear combustion. One-dimensional calculations show that the two possible modes of this combustion are either supersonic detonation or subsonic deflagration (Arnett 1969; Hansen \& Wheeler 1969; Ivanova et al. 1974; Nomoto et al. 1976; Nomoto et al. 1984). There is evidence, both theoretical and empirical, that SN Ia involve a transition from a deflagration to a detonation, or DDT (Khokhlov 1991a,b; Arnett \& Livne 1994; Höflich, Khokhlov \& Wheeler 1995; Höflich 1995; Wheeler et al. 1995; Höflich \& Khokhlov 1996 and references therein). Pure detonation models tend to produce an excess of iron peak elements, rather than the intermediate mass elements that are observed in the outer layers of SN Ia. Models in which DDT occurs can account for the properties of SN Ia, including the observed correlated dispersion in light curve amplitude and decay rate (Höflich et al. 1995; Höflich \& Khokhlov 1996). In such models, the burning front starts as a deflagration that makes a transition to a detonation directly (the standard "delayed detonation" model), or after the star has expanded due to partial burning and then recompressed (the "pulsation delayed detonation"). In these models, the density at which DDT occurs is a free parameter. The comparison of these models with observations suggests that DDT must

occur at relatively low densities, of the order of $10^{7} \mathrm{~g} \mathrm{~cm}^{-3}$. In this paper we address some of the key physical issues of DDT. In particular, we address the physical mechanism by which DDT may occur in supernovae, and why it might occur at low densities. The process of DDT is not well understood in either supernovae or in terrestrial combustion. Many of the principles presented here apply to terrestrial conditions as well.

The analysis we present is based on a theory of unconfined DDT (Khokhlov, Oran \& Wheeler 1996b). It is assumed that the inherent mechanism of DDT is the Zeldovich gradient mechanism (Zeldovich et al. 1970) in which ignition in a region of induction time 
gradient leads to the development of a reaction zone and a shock that coalesce to form a detonation. In this theory, it is presumed that a mixed region with gradients of temperature and composition, a combination of which results in an induction time gradient, is created within a deflagration region by turbulence that microscopically mixes hot products and cold fuel. Two quantities must be determined: the size of the critical region, $\mathrm{L}_{c}$, required for a self-propagating detonation to develop, and the amount of mixing (the intensity or velocity of turbulence) required to produce that critical length of mixing.

In supernovae, there are two situations in which DDT could occur. First, DDT could occur directly, when turbulent deflagration alone creates a large mixed region which ignites. For this process to occur, the flame surface associated with laminar burning has to be destroyed by turbulence. When the laminar flame front is destroyed, reactions will still continue at rates depending on the density and temperature, but microscopic chemical and thermal mixing of fuel and product occurs. The second way to initiate DDT is for the deflagration to die due to expansion before the DDT occurs. If insufficient energy has been liberated, the star will expand and then contract. Mixing of fuel and products, and the subsequent DDT may occur during the contraction phase.

In this paper, we first examine the mixing process and determine the thermodynamic properties of the mixture as a function of the degree of mixing. We show that these thermodynamic properties can be characterized by a single parameter (e.g., the mass fraction of fuel) and that the history of mixing, that is, whether mixing occurs before, after, or during a pulsation phase does not change the thermodynamic properties of the mixture significantly. We derive the critical length scale to initiate a detonation by the Zeldovich mechanism. Next, we evaluate the turbulent intensity necessary to break a laminar flame and to create the conditions for DDT. This required the calculation of the laminar flame speed and laminar flame thickness over a wide range of densities, $3 \times 10^{7}-10^{10} \mathrm{~g} \mathrm{~cm}^{-3}$. 
Finally, we estimate the critical density for DDT based on the existing three-dimensional simulations of turbulent deflagration in Type Ia supernovae, and discuss implications of the results for supernova models.

\section{Thermodynamics of mixing}

Consider a subsonic mixing process that occurs at approximately constant pressure, and assume that no reactions occur during mixing. The conservation of mass and enthalpy during mixing gives the thermodynamic parameters of the mixture,

$$
\begin{gathered}
h\left(T_{m}, \rho_{m}\right)=X_{f} h\left(T_{f}, \rho_{f}\right)+\left(1-X_{f}\right) h\left(T_{p}, \rho_{p}\right), \\
\frac{1}{\rho_{m}}=\frac{X_{f}}{\rho_{f}}+\frac{\left(1-X_{f}\right)}{\rho_{p}},
\end{gathered}
$$

where $h$ is the enthalpy; subscripts $f, p$, and $m$ stand for fuel, product, and mixture of products and fuel, respectively; $X_{f}$ is the fuel mass fraction in the mixture. We assume that the state of the products, $p$, is determined by conservation of enthalpy during constant pressure burning in a deflagration wave,

$$
\begin{gathered}
h\left(T_{p}, \rho_{p}\right)=h\left(T_{f}, \rho_{f}\right)+\Delta q, \\
P\left(T_{p}, \rho_{p}\right)=P\left(T_{f}, \rho_{f}\right)=\mathrm{const},
\end{gathered}
$$

where $\Delta q$ is the energy released by nuclear reactions.

The value of $\Delta q$ depends on the density at which the laminar flame is either quenched by turbulence or quenched by expansion, and is not known exactly. If the final stage of deflagration takes place at very low densities, say, less than $10^{7} \mathrm{~g} \mathrm{~cm}^{-3}$, only carbon would be able to react in a deflagration wave. Temperatures resulting from carbon burning would be so low that oxygen would not react. In this case, we expect $\Delta q \simeq 3.7 \times 10^{17} \mathrm{ergs}^{-1}$, and the products of burning would consist predominantly of half silicon and half oxygen by 
mass. At higher densities, both carbon and oxygen will burn to form silicon group elements. For the latter case, we pick a typical value of $\Delta q \simeq 6 \times 10^{17} \mathrm{ergs}^{-1}$. Below we refer to these two choices as Case A and B, respectively.

Now consider mixing and changes in pressure due to global adiabatic expansion or contraction. For a perfect gas, the order of mixing and expansion or contraction does not change the final state of mixed material. For the equation of state of white dwarf material, this is not an exact statement, but we show that it is a good approximation.

Figure 1 shows the results of the following numerical experiment using the degenerate matter equation of state described in the next section. A half carbon, half oxygen fuel at density $5 \times 10^{6} \mathrm{~g} \mathrm{~cm}^{-3}$ (point $f$ in Figure 1) was burned at constant pressure to a half silicon and half oxygen mixture (to reach point $p$ ) and then mixed with $X_{f}=0.5$ at constant pressure to yield the conditions at point $m_{o}$. Alternatively, both fuel and products were expanded adiabatically by a factor of 100 (to points $f^{\prime}, p^{\prime}$ ), and then mixed at constant pressure to produce the conditions at point $m^{\prime}$. Finally, the mixture was returned along the adiabat to the initial pressure. The final state, point $m$, is very close to $m_{o}$, even under this extreme adiabatic expansion and contraction. The difference in temperature between points $m$ and $m^{\prime}$ is $5 \%$, and the difference in density is $7 \%$. We conclude that, to a very good approximation, the thermodynamic properties of a mixture do not depend on the history of the mixing and expansion processes. We make that assumption in the following analysis and neglect the order in which mixing and expansion or contraction occur.

Mixing cold fuel and hot products raises the entropy of the mixture, so that the mixture will ignite at lower density than the fuel alone. For cases A and B, Figure 2 gives the density at which the energy-release rate in the fuel-product mixture is one-tenth of the maximum energy-release rate for isochoric burning at this density. The maximum release rate occurs after ignition, as the temperature rises and before fuel is consumed. Once the 
mixture is at this density, the induction time is very short, only ten times the characteristic time required to burn all of the fuel. The figure shows that for a pure fuel to ignite, it needs to be compressed to very high densities. However, even for fuel mixed with rather small amounts of hot products, the ignition density decreases drastically, and is $\simeq 10^{6}-10^{7}$

$\mathrm{g} \mathrm{cm}^{-3}$ for a wide range in fuel fraction. If matter is returning to high densities after a pulsation, a mixture containing a noticeable amount of products cannot be compressed to densities significantly in excess of $10^{7} \mathrm{~g} \mathrm{~cm}^{-3}$ before it ignites. For the pulsation delayed detonation models, therefore, the thermodynamic considerations place a limit on the conditions at which DDT can occur: DDT can only occur at densities near or below $10^{7} \mathrm{~g}$ $\mathrm{cm}^{-3}$. For the standard delayed detonation models, however, no such limit can be placed by thermodynamic considerations. For both cases, the kinetics and hydrodynamics of ignition need to be considered to determine whether DDT can occur at thermodynamically allowed conditions.

\section{Critical length scale for DDT}

We now determine the critical size of the mixed region capable of triggering a detonation. It has been shown (Khokhlov et al. 1996b) that there are two processes that are necessary for DDT to occur by the Zeldovich mechanism. First, a spontaneous wave must be generated and a shock-reaction complex must be formed. This complex forms somewhere in the middle of the mixed region where there is both enough fuel and high enough temperature. Second, this complex must be able to survive the propagation into the unburned material.

To estimate the critical size of this region, $\mathrm{L}_{c}$, we consider, as in Khokhlov et al. 1996b), a nonuniform region created by mixing the products of isobaric burning and fresh 
fuel, such that there is a linear spatial distribution of fuel fraction $X_{f}$,

$$
X_{f}(x)=\left\{\begin{array}{ll}
x / L, & 0 \leq x \leq L \\
1, & x>L
\end{array},\right.
$$

where $L$ is the size of the mixed region. This creates a region of oppositely directed gradients in temperature and concentration of reactants. Initially, the velocity of the material is zero, and the pressure $P_{0}$ is constant everywhere. The boundary conditions at $x=0$ are reflecting walls (symmetry conditions).

The system is described by the Euler equations coupled with the nuclear kinetic equations

$$
\begin{gathered}
\frac{\partial \rho}{\partial t}+\nabla \cdot(\rho \mathbf{U})=0 \\
\frac{\partial \rho \mathbf{U}}{\partial t}+\nabla \cdot(\rho \mathbf{U U})+\nabla P=0, \\
\frac{\partial E}{\partial t}+\nabla \cdot((E+P) \mathbf{U})=0, \\
\frac{\partial \mathcal{Y}}{\partial t}+(\mathbf{U} \cdot \nabla) \mathcal{Y}=\mathcal{R},
\end{gathered}
$$

where the total energy density $E=E_{t}+\rho U^{2} / 2+\rho N_{a} \mathcal{Q} \cdot \mathcal{Y}$ is the sum of the thermal, kinetic, and nuclear energies, $\mathbf{U}$ is the fluid velocity, $E_{t}$ is the thermal energy density, $\mathcal{Y}$ is the vector in the composition space of mole fractions of nuclei, $\mathcal{Q}$ is the vector of corresponding binding energies per nucleon, and $\mathcal{R}\left(\rho, E_{t}, \mathcal{Y}\right)$ is the vector of net rates of change of nuclear species with time; $N_{a}$ is the Avogadro number. To describe thermonuclear burning, an alpha-nuclei reaction network is used with $\mathcal{Y}=\left({ }^{4} \mathrm{He},{ }^{12} \mathrm{C},{ }^{16} \mathrm{O},{ }^{20} \mathrm{Ne},{ }^{24} \mathrm{Mg},{ }^{28} \mathrm{Si},{ }^{32} \mathrm{~S},{ }^{36} \mathrm{Ar},{ }^{40} \mathrm{Ca},{ }^{44} \mathrm{Ti},{ }^{48} \mathrm{Cr},{ }^{52} \mathrm{Fe},{ }^{56} \mathrm{Ni}\right)$. The equation of state includes contributions from Fermi gases of electrons and positrons, radiation, and ions. Further details of the input physics can be found in (Khokhlov 1993).

The system of equations is integrated numerically using a one-dimensional version of the time-dependent, compressible fluid code ALLA based on the Piecewise Parabolic 
Method (Collela \& Woodward 1984; Collela \& Glaz 1985), implemented as lagrangian step plus remap. Details of the implementation are given in (Khokhlov 1995; Khokhlov et al. 1996a). The nuclear reactions are coupled to fluid dynamics by time-step splitting. The kinetic equations are integrated together with the equation of energy conservation using a stiff solver with adjustable substeps to keep the accuracy better than $1 \%$. Most computations were done using a grid with 1024 equidistant computational cells. The convergence of the numerical solutions was tested for selected cases by varying the number of cells from 1024 to 4096. Computations were carried out in both planar and spherical geometries.

Computations were performed assuming that the products of burning that formed the mixture (state $p$ in the thermodynamic relations given in equations 1 to 4 ) are characterized by the value of the energy released by the deflagration $\Delta q=4 \times 10^{17}$ ergs $\mathrm{g}^{-1}$, or $\Delta q=3 \times 10^{17} \mathrm{ergs}^{-1}$. These values are close to Case $\mathrm{A}$ of $\S 2$ and have been selected to test the sensitivity of the results to $\Delta q$. For $\Delta q=4 \times 10^{17}$, the composition of products was taken to be pure ${ }^{28} \mathrm{Si}$. For the second value, the composition of products was taken to be half ${ }^{28} \mathrm{Si}$ and half ${ }^{16} \mathrm{O}$ by mass. The system was prepared in an initial state and then allowed to evolve in time, first until ignition takes place, then until the formation (or failure) of detonation, and finally to the time when the generated detonation or shock leaves the computational domain. The results of these computations are not sensitive to the assumed energy release and composition. They mainly depend on the density at which explosion occurs, and on the size of the explosion region.

Figure 3 is an example of a computation in which a detonation successfully forms. The initial density of the fuel $\rho_{0}=1.0 \times 10^{7} \mathrm{~g} \mathrm{~cm}^{-3}$ and the size of the mixed region is $L=5.0 \times 10^{5} \mathrm{~cm}$. Figures 3a through 3e show profiles of density, temperature, pressure, velocity, and carbon mole fraction, respectively, for selected times. In the initial state, the 
density and carbon mole fraction of the mixture increase, and the temperature decreases as a function of position $x$. Because of such opposing temperature and concentration gradients, the ignition does not occur first at the origin, $x=0$, but is shifted to some point $x>0$ where the energy generation rate is maximum. From this point, the ignition spreads out supersonically as a spontaneous wave. A detailed discussion of spontaneous burning is given in Khokhlov et al. (1996b). The overpressure and material velocity in the spontaneous wave grow with time as the phase velocity of the burning front decreases. When the speed of the spontaneous wave approaches the speed of sound, a shock wave emerges, and a shock wave - reaction complex forms. The profiles 1 to 3 illustrate the spontaneous wave propagation. There is no shock present at this stage. Matter is continuously compressed and accelerated in the process of burning, and expands and slows down afterwards. The shock - reaction complex forms between the profiles 3 and 4 . The complex continues to propagate through the matter in which density increases and temperature decreases (profiles 4 and 5), but the strength of the complex grows. Finally, the complex passes into the fully unburnt cold fuel in the form of a slightly overdriven detonation (profile 6), and begins its relaxation to a Chapman-Jouguet state.

Figures $3 \mathrm{f}$ and $3 \mathrm{~g}$ show the nuclear composition for the times corresponding to profiles 2 and 7 , respectively. These figures illustrate that at densities $\lesssim 10^{7} \mathrm{~g} \mathrm{~cm}^{-3}$, the important reactions are ${ }^{12} \mathrm{C}+{ }^{12} \mathrm{C} \rightarrow{ }^{20} \mathrm{Ne},{ }^{24} \mathrm{Mg},{ }^{4} \mathrm{He}$, and the reactions on ${ }^{20} \mathrm{Ne}$ and ${ }^{24} \mathrm{Mg}$ that transform the products of ${ }^{12} \mathrm{C}+{ }^{12} \mathrm{C}$ burning into the ${ }^{28} \mathrm{Si}$-group elements. Reactions involving oxygen are less important. The initial amount of oxygen in the mixture remains practically intact. The energy release is approximately $\Delta q \simeq(3.5-4) \times 10^{17} \mathrm{ergs} \mathrm{g}^{-1}$, consistent with the value of the energy release for the products of deflagration adopted above, and with the assumptions about the energy release made in $\S 2$. Figures $4 \mathrm{a}$ and $4 \mathrm{~b}$ show a successful initiation of a detonation at a density five times higher, $\rho_{0}=5 \times 10^{7}$ $\mathrm{g} \mathrm{cm}^{-3}$. At this density, burning leads to higher temperatures due to a decrease in the 
specific heat of degenerate matter. As a result, oxygen burns to silicon rather quickly (Fig.4b), and the energy of the explosion, $\Delta q \simeq 7 \times 10^{1} 7 \mathrm{ergs}^{-1}$, is almost two times larger than in the previous case $\rho_{0}=10^{7} \mathrm{~g} \mathrm{~cm}^{-3}$.

The shock-reaction complex can survive propagation down the temperature gradient and can grow into a fully developed detonation only if conditions in front of the complex change slowly enough, that is, if $L$ is large enough. If the variations of preshock density and temperature with distance are too fast, the reaction zone structure is unable to adjust to the decreasing reaction rates behind the shock, and is unable to compensate for the energy required to shock denser fuel.

Figures 5 a to $5 \mathrm{f}$ illustrate the failure of the initiation of a detonation at $\rho_{0}=10^{7} \mathrm{~g} \mathrm{~cm}^{-3}$ when the size of the mixed region was 20 percent smaller, $L=4 \times 10^{5} \mathrm{~cm}$, than in the case presented in Fig. 3. Here, too, the spontaneous wave, and the shock - reaction complex form (profiles 1 to 3). However, the complex does not survive the propagation down the temperature gradient. The shock and reaction separate. The shock passes into the unburned matter leaving the reaction wave behind. It is the condition that the complex must survive the propagation down the temperature gradient, not the formation of the complex alone, that determines the critical size of the mixed region.

Figure 6 summarizes the results of a series of many computations performed to determine $L_{c}$, the critical size of the region capable of triggering a detonation, as a function of fuel density. The value of $L_{c}$ is a very sensitive function of density. It is virtually independent of assumptions made about the energy release $\Delta q$ and composition of the products formed during the preceeding deflagration stage. The critical length depends, however, on the energy release during the explosion itself. As was mentioned earlier, the energy release increases with increasing background density, which makes the dependence of $L_{c}$ on density very strong. The values of $L_{c}$ obtained in spherical and planar geometries are 
practically the same, because the radius of curvature of a spherical front is approximately the same as the critical length which is much larger than the detonation wave thickness.

The simulations show that the spontaneous wave grows to an appreciable strength, and a shock - reaction complex forms in a part of the mixed region with a high fuel fraction, $X_{f} \simeq 0.8-0.9$. Thus, it is this mixture that is important for DDT. As shown in Figure 2, explosion of such a mixture can take place at densities $\leq 10^{7} \mathrm{~g} \mathrm{~cm}^{-3}$ (Fig. 2). On the other hand, as shown in Figure 6, the critical length for DDT is a sensitive function of density; it increases steeply at low densities and it is of order $3 \times 10^{7}-4 \times 10^{5} \mathrm{~cm}$ at densities of $5 \times 10^{6}-10^{7} \mathrm{~g} \mathrm{~cm}^{-3}$. This length is a factor of $10^{4}-10^{5}$ larger than the size of a detonation reaction zone. However, even at these low densities, this length is small compared to the radius of a white dwarf. The length scale $L_{c}$ is small enough to be formed by turbulent mixing in a relatively small portion of the supernova ejecta for $\rho \gtrsim 5 \times 10^{6} \mathrm{~g} \mathrm{~cm}^{-3}$.

\section{Conditions on turbulent intensity}

Here we discuss the conditions necessary to produce a mixed region of size $\mathrm{L}_{c}$ for both the direct and the pulsation modes of delayed detonation. First consider DDT in the direct mode. A fundamental assumption is that turbulence must destroy the flame front so that burned and unburned materials can mix microscopically. To break the flame front, the turbulence velocity $U_{b}$, at a scale comparable to the thickness of the laminar front, must exceed the normal speed of the laminar flame,

$$
U_{b} \simeq K S_{\ell}
$$

where $S_{\ell}$ is the laminar flame speed, $\delta_{\ell}$ is the laminar flame thickness, and $K \sim 1$ is a coefficient that describes the ability of the laminar flame to survive in a turbulent environment ( Khokhlov et al. 1996b). 
The mechanism for breaking the flame is flame stretching, which includes the effects of strain and curvature. These effects depend on the properties of the particular flame and on the spectrum of the turbulence. For terrestrial flames, the coefficient $K$ significantly exceeds unity according to both theory and experiments (Poinsot, Candel \& Trouvé 1996; Roberts et al. 1993). For the thermonuclear flame, the value of $K$ is not known exactly. The analysis of the nonlinear stabilization of the flames in a turbulent field suggests $K=8$ (Khokhlov 1995). The numerical simulations of the flame presented in (Khokhlov 1995) are consistent with a large value of $K$. For the subsequent analysis in this paper, we use the two values $K=1$ and $K=8$.

Figure 7 shows the laminar flame speed $S_{l}$ and thickness $\delta_{l}$ for a $0.5 \mathrm{C}+0.5 \mathrm{O}$ mixture computed for the range of densities $3 \times 10^{7}-10^{10} \mathrm{~g} \mathrm{~cm}^{-3}$, as described in the appendix. In the same figure, we show $S_{l}$ according to Timmes and Woosley (1992) who computed the laminar flame speed at high densities, $\rho \geq 2 \times 10^{8} \mathrm{~g} \mathrm{~cm}^{-3}$. Our results are in good agreement with theirs at these densities. At low densities the flame speed decreases with density more rapidly than at high densities, and reaches a very low value of $S_{l} \simeq 3 \times 10^{4}$ $\mathrm{cm} / \mathrm{s}$ at $\rho \simeq 3 \times 10^{7} \mathrm{~g} \mathrm{~cm}^{-3}$. The computed values of $S_{\ell}$ give $U_{b}$ assuming $K=1$. The curve $U_{b}^{8}=8 S_{l}$ shows the required turbulent intensity if $K=8$.

To relate $U_{b}$ to the intensity of turbulent motions on larger scales, additional assumptions are required about the spectrum of turbulent motions inside the white dwarf during the explosion. The most favorable assumption for DDT is that the turbulent spectrum is Kolmogorov. By assuming that a Kolmogorov spectrum is established, we ignore the time delay required to establish the steady-state cascade of turbulent energy, and ignore freeze-out of turbulence due to the expansion of the star. Under the assumption of a Kolmogorov spectrum, the speed of turbulent motions at the scale of the laminar front $\delta_{l}$ is 
related to the speed of turbulent motions on larger scales $L>\delta_{l}$ as

$$
U_{\delta_{l}}=U_{L}\left(\frac{\delta_{\ell}}{L}\right)^{1 / 3}
$$

During the deflagration phase of the explosion, large portions of the star will be occupied by the very convoluted flame. To be specific, we pick a typical value $L=L_{f} \simeq 10^{8}$ $\mathrm{cm}$ for the maximum size of the turbulent flame region (Khokhlov 1995). The turbulent region may be somewhat smaller in the beginning of the explosion, and somewhat larger at the end, but this value is of the correct order of magnitude. If we assume that the deflagration is strong enough to unbind the star, we have to assume that $L_{f}$ is large enough. The large-scale turbulence in a supernova is driven by buoyancy. If we neglect the effects of freeze-out due to expansion of the star, we expect $U_{L_{f}}$ to be of order of the Rayleigh-Taylor characteristic velocity at this scale $U_{L_{f}} \simeq U_{R-T} \simeq 0.5 \sqrt{g L_{f}}$ where $g$ is the effective gravitational acceleration. In a star in hydrostatic equilibrium, or close to it, the value of $\sqrt{g L_{f}}$ does not exceed the speed of sound. In equilibrium, $P / R \sim \rho g$, where $\mathrm{R}$ is of order the stellar radius. From this it follows that $a_{s} \simeq \sqrt{P / \rho} \sim \sqrt{g R}$ and hence $\sqrt{g L_{f}}<a_{s}$ for $L_{f}<R$. For our estimates we take the value of $U_{L_{f}}=U_{R-T}=0.5 a_{s}$. The values of the turbulent velocity at the scale of the flame front estimated from equation (11) using these values is plotted in Figure 7 . The intersection of the curve $U_{\delta_{l}}$ with the curves $S_{l}$ and $U_{b}^{8}$ gives estimates of the transition density $\rho_{\mathrm{tr}} \leq(4-8) \times 10^{7} \mathrm{~g} \mathrm{~cm}^{-3}$ below which DDT may occur. We emphasize that this estimate of $\rho_{\text {tr }}$ is made neglecting any time delay in establishing the Kolmogorov spectrum, and neglecting freeze-out of turbulence due to expansion of the star.

In order to take the effect of freeze-out into account, three-dimensional simulations of the explosion of the entire star are required. To date, only one such simulation has been performed (Khokhlov 1995), and the analysis presented in that paper suggests that the expansion freezes scales exceeding approximately $L \simeq 10^{6}-10^{7} \mathrm{~cm}$, and that the turbulent 
burning velocity at these scales does not exceed $U_{L} \simeq 10^{6}-10^{7} \mathrm{~cm} / \mathrm{s}$. If we take from this range the most favorable values for DDT, $U_{L}=10^{7} \mathrm{~cm} / \mathrm{s}$ and $L=10^{6} \mathrm{~cm}$, equation (11) gives another estimate of the turbulent velocity at the scale of the flame front, $U_{\delta_{l}}^{c}$, which is also shown in Figure 7. The intersection of the curve $U_{\delta_{l}}^{c}$ with the curves $U_{b}^{8}$ and $S_{l}$ gives, we believe, a more realistic estimate of the transition density $\rho_{\operatorname{tr}} \leq(2-5) \times 10^{7} \mathrm{~g} \mathrm{~cm}^{-3}$.

There are other effects that may influence the transition density. Self-turbulization of the flame on scales less than $L_{f}$ might be possible, as is preconditioning of a mixture by shocks generated by the turbulence itself. The latter effects might somewhat facilitate DDT, but not to a great extent (Khokhlov et al. 1996b). Self-turbulization due to the Landau-Darrieus instability is limited by nonlinear effects. Shock preconditioning requires a very strong shock to be effective. We believe that there are two major unknown factors in estimating the value of $\rho_{\mathrm{tr}}$ : the spectrum of turbulence in the exploding star, and the extent to which the flame can survive turbulent stretch on small scales (coefficient $K$ ).

Now consider the case in which the conditions for DDT do not occur in the deflagration phase, and the deflagration is extinguished by the expansion. In this case, burning is quenched by expansion and the products and fuel mix freely. There is no laminar flame front and there are no nuclear reactions. This situation is still Rayleigh-Taylor unstable. Previous estimates (Khokhlov 1991a; Arnett \& Livne 1994) have shown that the mixed $\mathrm{R}-\mathrm{T}$ region may be on the order of $10^{6}-10^{8} \mathrm{~cm}$, which is much larger than $L_{c}$ at $\rho \gtrsim 5 \times 10^{6}-10^{7} \mathrm{~g} \mathrm{~cm}^{-3}$. With a Kolmogorov cascade, the eddy turnover time scales as $\lambda^{2 / 3}$, where $\lambda$ is the size of the eddy. When the star is compressed, the turbulent motions on all scales will be enhanced and mixing should proceed vigorously (this is opposite to the effect of freeze-out expected during the expansion of the star). It is thus reasonable to expect microscopic mixing to be complete before the contraction phase.1. This suggests that

\footnotetext{
${ }^{1}$ We have invoked microscopic mixing in this model. There is considerable evidence that
} 
the pulsation mechanism can lead rather naturally to conditions where DDT is unavoidable in the recompression phase.

\section{Conclusions}

We applied a theory of DDT in unconfined conditions (Khokhlov et al. 1996a) to DDT in Type Ia supernovae. The two basic assumptions of this theory are: 1) the gradient mechanism is the inherent mechanism that leads to DDT in unconfined conditions, and 2) the mechanism for preparing the gradient of induction time is turbulent mixing that requires breaking or quenching the flame front.

Using a series of numerical simulations, we determined the minimum critical size of the mixed region, $L_{c}$, required for DDT $(\S 3)$. We find that $L_{c}$, though much larger than the detonation wave thickness, is at least two to three orders of magnitude smaller than a white dwarf radius for $\rho>5 \times 10^{6}-10^{7} \mathrm{~g} \mathrm{~cm}^{-3}$. Thus, a mixed region of critical size required for triggering a detonation can, in principle, be formed by turbulent mixing in a small portion of a supernova.

There are two possible modes of detonation formation in a Type Ia supernova: a direct

mixing occurs in supernova ejecta but is not always microscopic. Several lines of evidence strongly suggest that the mixing in SN 1987A was macroscopic, but not microscopic. We note, however, that the physical conditions here and in a collapse environment like SN 1987A are different. The instability leading to mixing in SN 1987A is expected to be a RichtmeyerMeshkov instability in which the density/pressure inversion is only temporarily induced at composition/density boundaries due to shock passage. In the thermonuclear explosions that we consider here, the Rayleigh-Taylor instability is intrinsic and long lasting. There should be ample time to mix to small length scales. 
mode when DDT occurs inside an active deflagration front, and a pulsation mode which is possible if DDT does not occur directly, and if the energy released during the deflagration phase is not enough to unbind the star. Then the star will experience a global pulsation, and mixing will take place between the cold fuel and ashes of the dead deflagration. The requirements on the intensity of turbulent mixing needed to form a mixed region of critical size $L_{c}$ are different in these two cases.

For a direct mode of DDT (delayed detonation model), the intensity of turbulence on small scales of the order of the laminar flame thickness must be high enough to stretch and break the surface of the laminar flame front ( $\S 4$, equation 10). We computed the laminar flame velocity and thickness in a wide range of densities $3 \times 10^{7}-10^{10} \mathrm{~g} \mathrm{~cm}^{-3}$, and estimated the critical intensity of turbulence required to break the flame at the scale of a laminar flame thickness.

We also tried to estimate the value of the transition density $\rho_{\text {tr }}$ at which DDT should occur during a Type Ia supernova explosion. Using available information about the intensity of turbulence inside the exploding star, we conclude that a realistic estimate of the transition density for the direct delayed detonation explosion is $\rho_{\mathrm{tr}} \lesssim(2-5) \times 10^{7} \mathrm{~g} \mathrm{~cm}^{-3}$. However, even under the most favorable and unrealistic assumptions $(\S 4), \rho_{\text {tr }}$ does not exceed $10^{8} \mathrm{~g} \mathrm{~cm}^{-3}$.

In the pulsation mode (pulsating delayed detonation model), microscopic mixing throughout large areas can occur because there is no flame front separating burned and unburned matter, and, thus, fuel and products can mix freely. We considered the thermodynamics of the mixing process in a supernova $(\S 2)$ to demonstrate that the order of mixing and global expansion or contraction of the supernova is not important. We also found that even a small fraction $(\gtrsim 1 \%)$ of high-entropy products of burning mixed with cold, low-entropy fuel increases the entropy of the mixture such that it will ignite at very 
low densities. We conclude that in this mode, the portions of the mixture of size comparable to or exceeding $L_{c}$ would unavoidably detonate around $\rho_{\mathrm{tr}} \simeq 10^{7} \mathrm{~g} \mathrm{~cm}^{-3}$.

Despite various details of DDT that require future consideration, our main conclusion is that DDT in Type Ia supernovae should occur in the density range $5 \times 10^{6}$ to a few times $10^{7}$. At higher densities, $10^{8}-10^{9} \mathrm{~g} \mathrm{~cm}^{-3}$, either the required turbulent velocity cannot be reached (direct mode of the delayed detonation) or the material inevitably ignites before such high densities are reached (pulsating mode of the delayed detonation). At lower densities, the size of the critical region becomes too large. This conclusion agrees with the results of global modeling of Type Ia supernovae, where the transition density, $\rho_{\mathrm{tr}}$, at which DDT occurs is a free parameter. Global modeling suggests that, in order to fit observed light curves and spectra of SNIa, the transition density should be $\rho_{\mathrm{tr}} \simeq 10^{7} \mathrm{~g} \mathrm{~cm}^{-3}$ (Khokhlov 1991a; Woosley \& Weaver 1994; Höflich et al. 1995; Nomoto et al. 1995; Höflich 1995; Höflich \& Khokhlov 1996).

This work was supported by NSF grants 92-18305 and 94-17083, NASA grant NAG52888, Texas Advanced Research Program grant ARP 469, and by the Naval Research Laboratory through the Office of Naval Research. We thank P.A. Höflich for many important discussions and F.-K. Thielemann for providing the thermonuclear reaction rates. Input for the electron-conductivity calculations was kindly provided by D. Yakovlev. Peter Hauschildt and the anonymous referee made very useful comments that led to improvements of the paper during the refereeing process. 


\section{A. Properties of laminar flames in degenerate $\mathrm{C}-\mathrm{O}$ mixtures}

The velocity of a laminar flame $S_{l}$ is computed as the eigenvalue of the differential equations describing the structure of a steady-state burning wave (Zeldovich et al. 1985). We assume that the wave propagates steadily and that burning occurs at constant pressure since the flame speed is much less than the sound speed. The parameters of the fuel and the products of burning are designated below by subscripts 0 and 1, respectively. The mass flux is constant through a steady wave, $J_{m}=\rho u=\rho_{0} S_{l}=\rho_{1} u_{1}$. The equation of energy balance inside the wave is

$$
J_{m} c_{P} \frac{d T}{d x}=\frac{d}{d x}\left(\chi \frac{d T}{d x}\right)+J_{m} N_{a} \mathcal{Q} \cdot \mathcal{R},
$$

where $c_{P}$ is the specific heat at constant pressure, $\chi$ is the thermal conductivity, $N_{a}$ is the Avogadro number, $\mathcal{Q}$ is the vector of binding energies of nuclei, $\mathcal{R}$ is the vector of net

reaction rates (Section 3), and $x$ is the distance across the wave. The terms on the right side of (A.1) describe the temperature changes due to the heat conduction and nuclear reactions. Nuclear mole fractions $\mathcal{Y}$ are changing inside the wave due to nuclear reactions,

$$
J_{m} \frac{d \mathcal{Y}}{d x}=\rho \mathcal{R}
$$

Ion diffusion is ignored in (A.2) since, in a degenerate gas, the mean free paths of ions are much smaller than the mean free paths of electrons and photons. The density $\rho(T, \mathcal{Y})$ inside the wave is related to $T$ and $\mathcal{Y}$ via the constant pressure condition

$$
P(\rho, T, \mathcal{Y})=P_{0}
$$

The boundary conditions for Eqs.(A.1) to (A.3) are

$$
\text { at } x=-\infty: T=T_{0}, \rho=\rho_{0}, \mathcal{Y}=\mathcal{Y}_{0}, \frac{d T}{d x}=0
$$

and

$$
\text { at } x=\infty: \quad T=T_{1}, \rho=\rho_{1}, \mathcal{Y}=\mathcal{Y}_{1}, \frac{d T}{d x}=0
$$


The points $x= \pm \infty$ are singular points of equations (A.1)-(A.3). The integral curve which passes through these singular points represents the solution for a steady flame front. We designate the value of $J_{m}$ corresponding to this integral curve as $J^{*}$.

For low temperatures, $T<T_{\varepsilon}$, where $T_{\varepsilon}$ is some value less than $2 \times 10^{9} \mathrm{~K}$, we can neglect the energy generation term in (A.1) in comparison with the term describing the heat transport. At these temperatures, (A.1) can be rewritten as

$$
\frac{d W}{d T}=\frac{J_{m} c_{P}}{\chi}
$$

where $W=\chi \frac{d T}{d x}$. The corresponding initial condition for $(\mathrm{A} .7)$ is $W\left(T_{0}\right)=0$. The integration of (A.7) from $T_{0}$ to $T_{\varepsilon}$ gives a certain value of $W_{\varepsilon}=W\left(T_{\varepsilon}\right)$, from which we find the corresponding value of $\left(\frac{d T}{d x}\right)_{T_{\varepsilon}}$. From this point, we integrate the full equation (A.1) with the energy generation term included.

The value $J^{*}$ is found using the method of trial and error (Zeldovich et al. 1985). The integral curves for $J_{m} \neq J^{*}$ diverge from the $J^{*}$ curve when $x \rightarrow \infty$. For $J_{m}<J^{*}$ the asymptotic $(x \rightarrow \infty)$ behavior is $T \rightarrow-\infty, \frac{d T}{d x} \rightarrow-\infty$. For $J_{m}>J^{*}$ the asymptotic behavior is $T \rightarrow \infty, \frac{d T}{d x} \rightarrow \infty$. We use the difference in the behavior of the integral curves for discriminating between the cases $J_{m}<J^{*}$ and $J_{m}>J^{*}$. The next trial value of $J_{m}$ is found as the average of the two most recent trial values lying on different sides of $J^{*}$. It requires $\simeq 20$ iterations to obtain the value of $J^{*}$ with an accuracy of $10^{-3}$. Then the laminar flame speed is calculated as $S_{l}=J^{*} / \rho_{0}$. The values of $\delta_{l}$ are estimated approximately as the thickness of a zone where most of the nuclear energy is released and the CO mixture is converted into Si-peak elements. Computations are done for the initial mixture of equal masses of ${ }^{12} \mathrm{C}$ and ${ }^{16} \mathrm{O}$ with a small amount of ${ }^{22} \mathrm{Ne}$ added in order to get a neutron excess $\eta=0.002$. The initial temperature is $T_{0}=10^{8} \mathrm{~K}$, and we used $T_{\varepsilon}=10^{9}$ K. Flame parameters are not sensitive to variations of $T_{0}$ and $T_{\varepsilon}$ if these temperatures are below $2 \times 10^{9} \mathrm{~K}$. 
In computations of $S_{l}$ and $\delta_{l}$, the following 133 species reaction network is used: $n$, $p,{ }^{4} \mathrm{He},{ }^{12,13} \mathrm{C},{ }^{13} \mathrm{~N},{ }^{16} \mathrm{O},{ }^{20,22} \mathrm{Ne},{ }^{23} \mathrm{Na},{ }^{23-26} \mathrm{Mg},{ }^{27} \mathrm{Al},{ }^{27-32} \mathrm{Si},{ }^{30-33} \mathrm{P},{ }^{31-36} \mathrm{~S},{ }^{35-37} \mathrm{Cl}$, ${ }^{36-41} \mathrm{Ar},{ }^{39-43} \mathrm{~K},{ }^{40-46} \mathrm{Ca},{ }^{43-47} \mathrm{Sc},{ }^{44-50} \mathrm{Ti},{ }^{47-52} \mathrm{~V},{ }^{48-56} \mathrm{Cr},{ }^{51-60} \mathrm{Mn},{ }^{52-62} \mathrm{Fe},{ }^{55-61} \mathrm{Co}$, ${ }^{56-64} \mathrm{Ni},{ }^{57-65} \mathrm{Cu},{ }^{59-66} \mathrm{Zn}$. The forward reaction rates are taken from Thielemann's reaction rate library (Thielemann, Arnould \& Truran 1987). The binding energies and partition functions were taken according to Woosley et al. (1987). The backward reaction rates are calculated according to the principle of detailed balance. Screening corrections are applied to the most important reactions, ${ }^{12} \mathrm{C}+{ }^{12} \mathrm{C},{ }^{12} \mathrm{C}+{ }^{16} \mathrm{O},{ }^{16} \mathrm{O}+{ }^{16} \mathrm{O}$ and $3{ }^{4} \mathrm{He} \rightarrow{ }^{12} \mathrm{C}$, which control the thermonuclear energy release at low temperatures. Even for these reactions screening factors were found to be relatively small $(E \lesssim 2-5)$ for conditions where nuclear reactions significantly contribute to the right side of Eq.(2). Most of the nuclear reactions occur at high temperatures and the corresponding screening factors $E \lesssim 1.5$ are small in comparison with the expected uncertainties in the nuclear reaction rates $(\sim 2-3)$. Screening corrections are taken from Yakovlev and Shalybkov (1989).

The total thermal conductivity is calculated as the sum of electron and photon conductivities

$$
\chi=\chi_{e}+\chi_{\gamma}
$$

with $\chi_{\gamma}=4 a c T^{3} / 3 \kappa \rho$. The expression for the photon opacity (Paczynski 1983)

$$
\kappa=\frac{0.4 Y_{e}}{\left(1+2.710^{11} \rho / T^{2}\right)}\left(1+\left(\frac{T}{4.510^{8}}\right)^{0.86}\right)^{-1} \frac{\mathrm{cm}^{2}}{\mathrm{~g}}
$$

approximates the low density $\left(\lesssim 610^{7} \mathrm{~g} \mathrm{~cm}^{-3}\right.$ ) results of Buchler and Yueh (1976) that take the degeneracy of electrons and relativistic scattering into account. $Y_{e}$ is the electron mole fraction. At higher densities, the contribution of the photon opacity to the total opacity is very small. Our results at high densities are in a good agreement with those of Timmes \& Woosley (1992) who used different approximations for the photon thermal conductivity.

The electron thermal conductivity can be expressed in terms of the effective electron 
collisional frequency, $\nu_{e}$, as (Yakovlev and Urpin 1980)

$$
\chi_{e}=4.0910^{9} \mathrm{~T} \frac{x^{3}}{\sqrt{1+x^{2}}}\left(\frac{10^{16}}{\nu_{e}}\right) \frac{\operatorname{ergs}}{\mathrm{cm} \mathrm{s} \mathrm{K}}
$$

where $x=p_{\mathrm{F}} / m_{e} c \simeq 1.00910^{-2}\left(\rho Y_{e}\right)^{1 / 3}, p_{\mathrm{F}}$ is the electron Fermi momentum, and $\nu_{e}$ is a sum of ion-electron and electron-electron collisional frequencies,

$$
\nu_{e}=\nu_{e i}+\nu_{e e}
$$

The electron - ion collisional frequency is calculated using the mean ion approximation following Urpin \& Yakovlev (1980)

$$
\nu_{e i}=1.7810^{16} \sqrt{1+x^{2}} \frac{Y Z^{2} \Lambda}{Y_{e}},
$$

where $Y=\sum Y_{i}$ and $Z=Y_{e} / Y$ are the mean ion mole fraction and the mean atomic number, respectively, and $\Lambda$ is the Coulomb logarithm which can be expressed for gaseous and liquid ionic states as the sum of the logarithm obtained in the Born approximation and the non-Born correction (Yakovlev and Urpin 1980, Yakovlev 1987)

$$
\Lambda=\Lambda_{\mathrm{B}}+\delta \Lambda,
$$

with

$$
\begin{gathered}
\Lambda_{\mathrm{B}}=\ln \left(\left(\frac{2 \pi Z}{3}\right)^{1 / 3} \sqrt{1.5+\frac{3}{\Gamma}}\right)-\frac{x^{2}}{2\left(1+x^{2}\right)}, \\
\Gamma=2.27510^{5} \frac{Z^{5 / 3}}{T}\left(\rho Y_{e}\right)^{1 / 3}
\end{gathered}
$$

and

$$
\begin{gathered}
\delta \Lambda=\frac{\pi}{2} \alpha \beta^{2} \frac{1+1.30 \alpha}{1+\alpha^{2}\left(0.71-0.54 \beta^{2}\right)}, \\
\beta=\frac{x}{\sqrt{1+x^{2}}} \quad, \quad \alpha=\frac{Z}{137 \beta} .
\end{gathered}
$$

The thermal conductivity due to electron-electron collisions of relativistic electrons in a degenerate electron gas was calculated in the static screening approximation by Urpin 
and Yakovlev (1980),

$$
\nu_{e e}=0.511 T^{2} \frac{x^{3 / 2}}{\left(1+x^{2}\right)^{5 / 4}} J(y) \mathrm{s}^{-1}
$$

where

$$
y=\frac{\sqrt{3} T_{p e}}{T},
$$

and

$$
T_{p e}=\frac{\hbar \omega_{p e}}{k_{\mathrm{B}}}=3.30710^{8} \frac{x^{3 / 2}}{\left(1+x^{2}\right)^{1 / 4}} \mathrm{~K}
$$

is the electron plasma temperature. Recently Yakovlev (unpublished) recalculated the integral entering the general expression of $\nu_{e e}$ numerically and obtained the following analytical expression for $J(y)$

$$
J(y)=\frac{1}{3}\left(\frac{y}{1+a y}\right)^{3} \ln \left(\frac{2}{y}+b\right)
$$

with $a=0.113$ and $b=1.247$. The largest error in the fit is $\sim 6 \%$ at $y \simeq 2$. 


\section{REFERENCES}

Arnett, W.D., 1969. Ap\&SS, 5, 180.

Arnett, W.D. \& Livne, E., 1994, ApJ, 427, 314.

Buchler J.R. \& Yueh W.R., 1976, ApJ, 210, 440.

Colella, P., and Woodward, P.R., 1984. Journ. Comp. Phys., 54, 174.

Colella, P., and Glaz, H.M., 1985. Journ. Comp. Phys., 59, 264.

Hansen J.P., Torrie G.M. \& Vieillefosse P., 1977, Phys. Rev., A16, 2153.

Hansen, C.J. \& Wheeler, J.C., 1969. Ap\&SS, 3, 646.

Höflich, P., 1995. ApJ, 443, 89.

Höflich, P., Khokhlov, A.M., \& Wheeler, J.C., 1995, ApJ, 444, 831

Höflich, P. \& Khokhlov, A.M., 1996. ApJ, 457, 500.

Ivanova, Imshennik, V.S. \& Chechetkin, V.M., 1974, Ap\&SS, 31, 497.

Khokhlov, A.M. 1991a, A\&A, 245, 114.

Khokhlov, A.M. 1991b, A\&A, 245, L25.

Khokhlov, A.M., 1993. ApJ, 419, 200.

Khokhlov, A.M., 1995. ApJ, 449, 695.

Khokhlov, A.M. Oran, E.S. \& Wheeler, J.C., 1996a, Combustion \& Flame, 105, 28.

Khokhlov, A.M. Oran, E.S. \& Wheeler, J.C., 1996b, Combustion \& Flame, in press.

Nomoto, K., Sugimoto, D. \& Neo, S., 1976. Ap\&SS, 39, L37. 
Nomoto, K., Thielemann, F.-K. \& Yokoi, K., 1984. ApJ, 286, 644.

Nomoto, K., Yamaoka, H., Shigeyama, T., Iwamoto, K., 1995. in Supernovae and Supernova Remnants, ed. R. McCray and Z. Wang, proc. of the IAU Colloquium 145 (Cambridge Univ. Press), p.

Paczynski B., 1983, ApJ, 267, 315.

Poinsot, T., Candel, S., \& Trouvé, A., 1996, Prog. Energy Combust. Sci., 21, 531.

Roberts, W.L., Driscoll, J.F., Drake, M.C., \& Goss, L.P., 1993, Combust. Flame, 94, 58.

Thielemann, F.-K., Arnould, M., Truran, J.W., 1987, Advances in Nulcear Astrophysics, ed. E. Vangioni-Flam (Editions frontières: Gif sur Yvette), p. 525.

Timmes, F. \& Woosley, S.E., 1992, ApJ, 396, 649.

Urpin V.A. \& Yakovlev D.G., 1980, Sov. Astron., 24, 126.

Wheeler, J.C, Harkness, R.P., Khokhlov, A.M. \& Höflich, P.A., 1995, Physics Reports, 256, 211.

Woosley, S.E., Holmes, J.A., Fowler, W.A. and Zimmerman, B.A., 1987, Atomic Data and Nuclear Data Tables, 22, 371.

Woosley, S.E. \& Weaver, T.A. 1994. in Supernovae, ed. S. Bludman, R. Mochkovitch \& J. Zinn-Justin (Les Houches Vol. 24: Elsevier), p.

Yakovlev D.G., 1987, Sov. Astron., 31, 347.

Yakovlev D.G. \& Shalybkov D.A., 1989, Sov. Sci. Rev. E., 7, 311.

Yakovlev D.G. \& Urpin V.A., 1980, Sov. Astron., 24, 303. 
Zeldovich, Ya.B., Librovich, V.B., Makhviladze, G.M. \& Sivashinsky, G.I, 1970, Astronaut. Acta 15, 313.

Zeldovich, Ya.B., Barenblatt, G.I., Librovich, V.B., Makhviladze, G.M., 1985, The Mathematical Theory of Combustion and Explosions (Consultants Bureau: London). 
Fig. 1 - Comparison of thermodynamic properties of mixtures $m$ and $m_{0}$ formed by mixing the same products and fuel at high and at low pressure, respectively.

Fig. 2 - Ignition density as a function of the fuel fraction. (Solid line) $-\Delta q=3.7 \times 10^{17}$ $\operatorname{ergs} \mathrm{g}^{-1} ;($ Dashed line $)-\Delta q=6.8 \times 10^{17} \operatorname{ergs~}^{-1}$.

Fig. 3 - Initiation of a detonation for $\rho_{0}=10^{7} \mathrm{~g} \mathrm{~cm}^{-3}$ and $L=10^{5} \mathrm{~cm}$. Profiles are shown for the initial time $t=0$, and for times $t=2.86 \times 10^{-5}(1), 7.05 \times 10^{-5}(2)$, $1.40 \times 10^{-4}(3), 1.63 \times 10^{-4}(4), 1.90 \times 10^{-4}(5), 2.33 \times 10^{-4}(6), 2.76 \times 10^{-4}(7)$ and $3.27 \times 10^{-4}$ (8) sec. (a) Pressure. (b) Temperature. (c) Density. (d) Velocity. (e) Carbon mole fraction. (f) Distribution of nuclear species at the time corresponding to profile 2. (g) Distribution of nuclear species at the time corresponding to profile 7 .

Fig. 4 - Initiation of a detonation for $\rho_{0}=5 \times 10^{7} \mathrm{~g} \mathrm{~cm}^{-3}$ and $L=1.3 \times 10^{3} \mathrm{~cm}$. Profiles are shown for the initial time $t=0$, and for the moments of time $t=1.74 \times 10^{-8}$ (1), $7.89 \times 10^{-8}(2), 1.38 \times 10^{-7}(3), 1.90 \times 10^{-7}(4), 2.41 \times 10^{-7}(5), 3.38 \times 10^{-7}(6)$, $4.31 \times 10^{-7}(7)$ and $4.96 \times 10^{-7}$ (8) sec. (a) Pressure. (b) Distribution of nuclear species at the time corresponding to profile 7 .

Fig. 5 - Failed initiation of a detonation for $\rho_{0}=1 \times 10^{7} \mathrm{~g} \mathrm{~cm}^{-3}$ and $L=8 \times 10^{4} \mathrm{~cm}$. Profiles are shown for the initial time $t=0$, and for the moments of time $t=5.64 \times 10^{-5}$ (1) $, 1.12 \times 10^{-4}(2), 1.68 \times 10^{-4}(3), 2.22 \times 10^{-4}(4), 2.77 \times 10^{-4}(5)$ and $3.31 \times 10^{-4}(6)$ sec. (a) Pressure. (b) Temperature. (c) Density. (d) Velocity. (e) Carbon mole fraction. (f) Distribution of nuclear species at the time corresponding to profile 5 .

Fig. 6 - Critical length for the initiation of a detonation as a function of the fuel density. (Circles) $-\Delta q=4 \times 10^{17} \mathrm{ergs}^{-1}$ for deflagration products. (Triangles) $\Delta q=3 \times 10^{17} \mathrm{ergs}^{-1}$ for deflagration products (all but one triangle coincide with the circles). 
Fig. 7 - Velocities and flame thickness as a function of density. $S_{l}$ and $\delta_{l}$ - laminar flame speed and thickness, computed in the appendix. $S_{l}^{T W}$ - laminar flame speed according to Timmes \& Woosley (1992). $U_{b}^{8}$ - critical turbulent velocity assuming $K=8 . U_{\delta_{l}}-$ estimated turbulent velocity at the scale of the flame front during the supernova explosion, assuming no turbulence freeze-out. $U_{\delta_{l}}^{c}$ - same, but assuming turbulence freeze-out. 


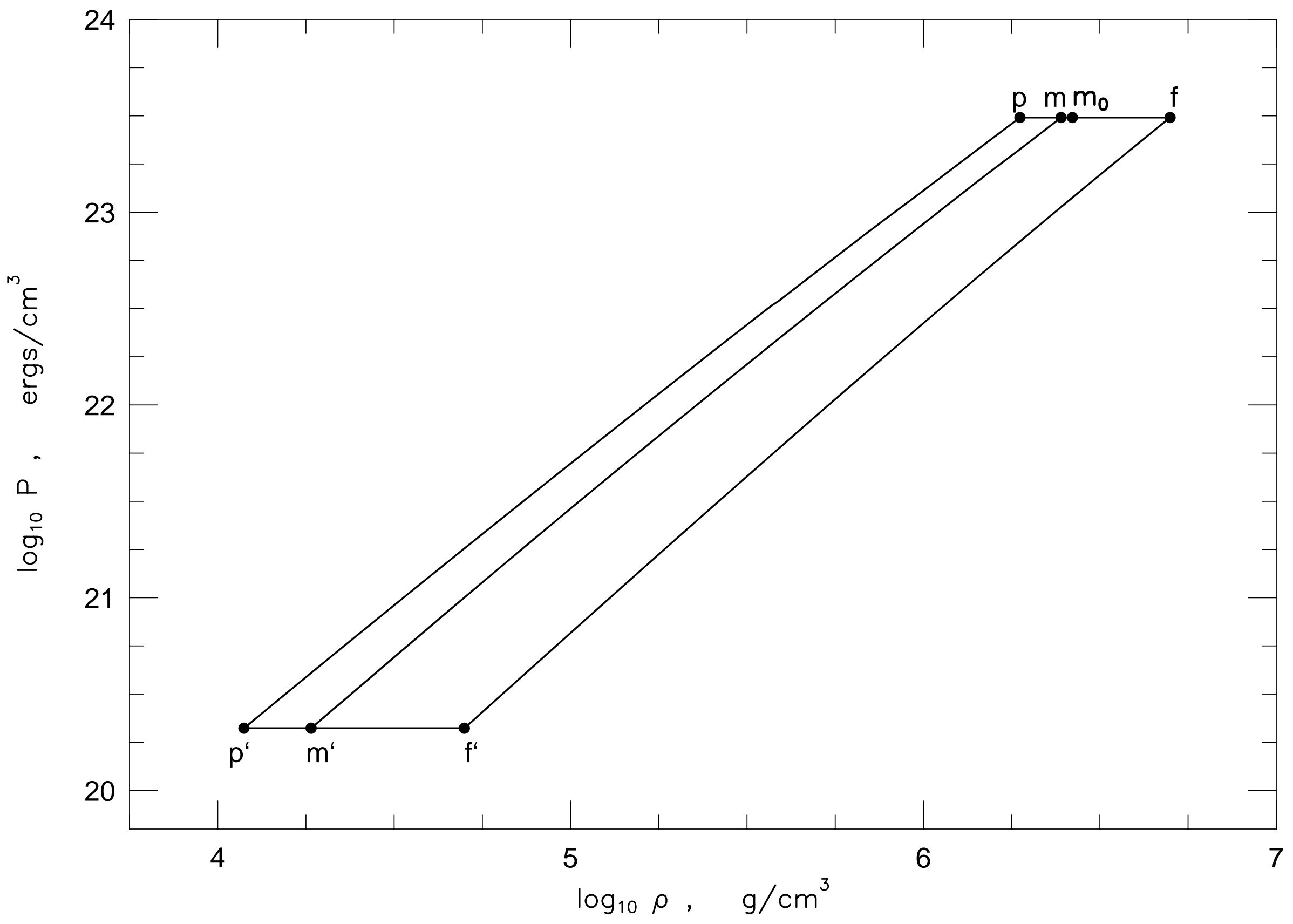




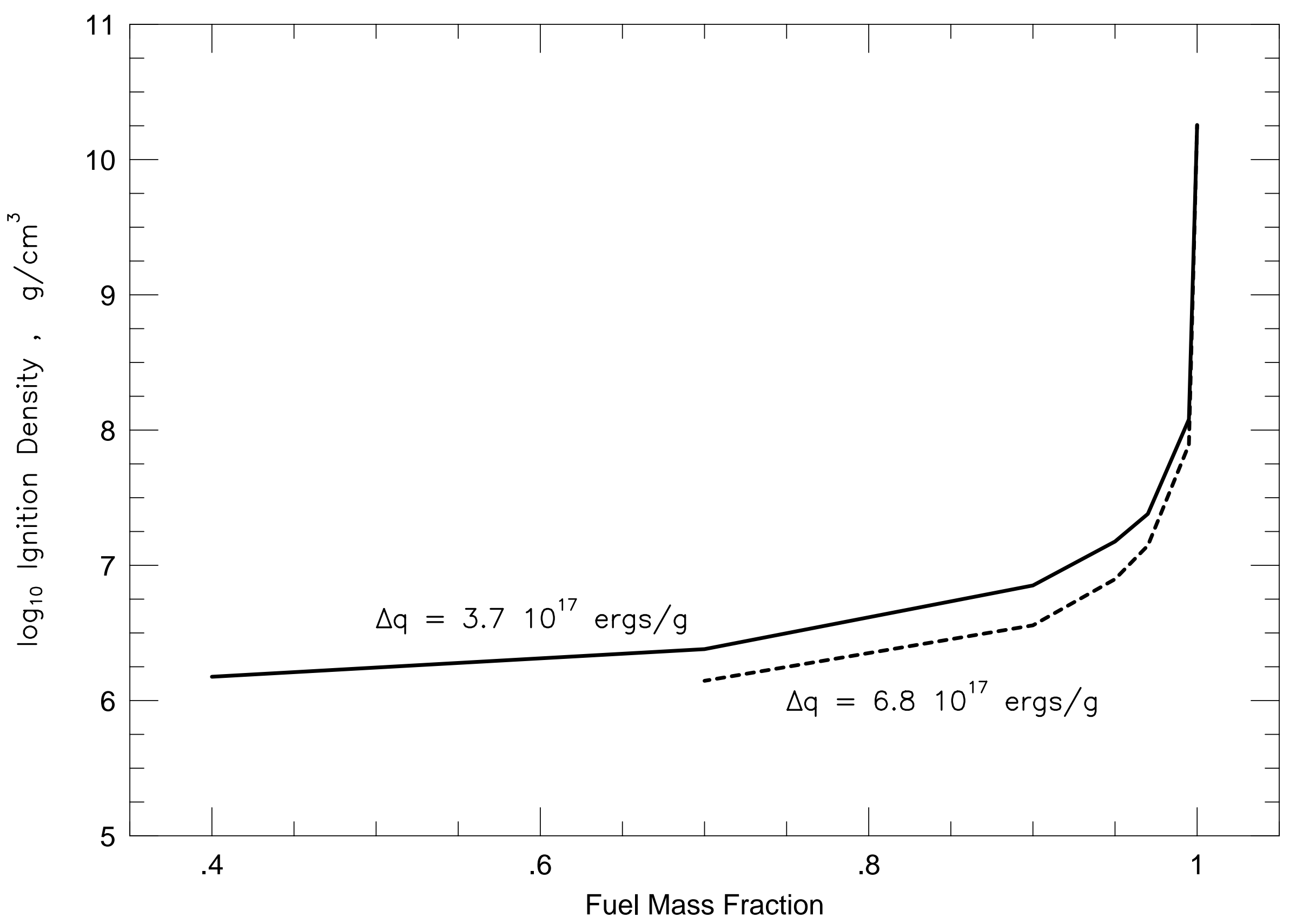




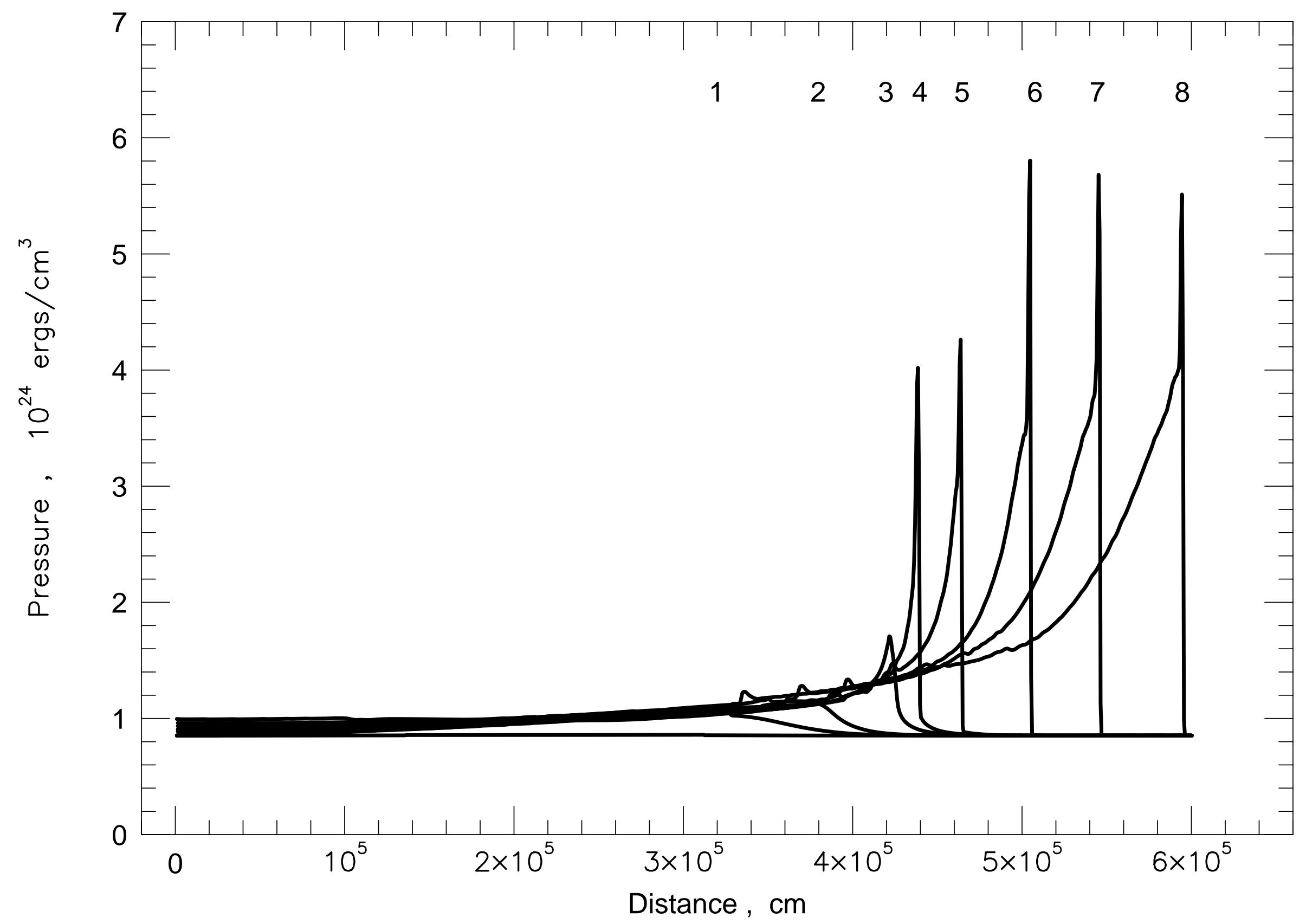




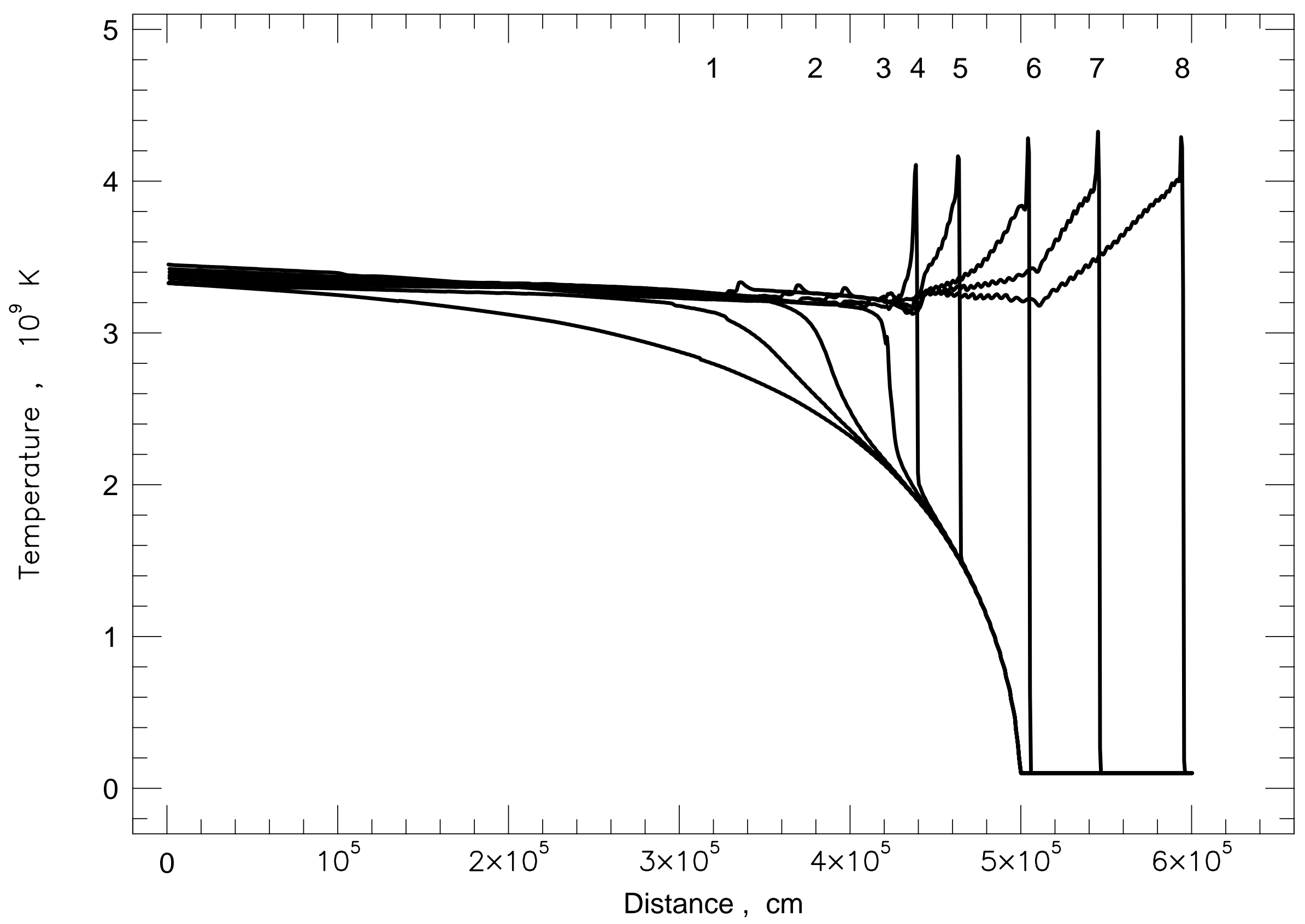




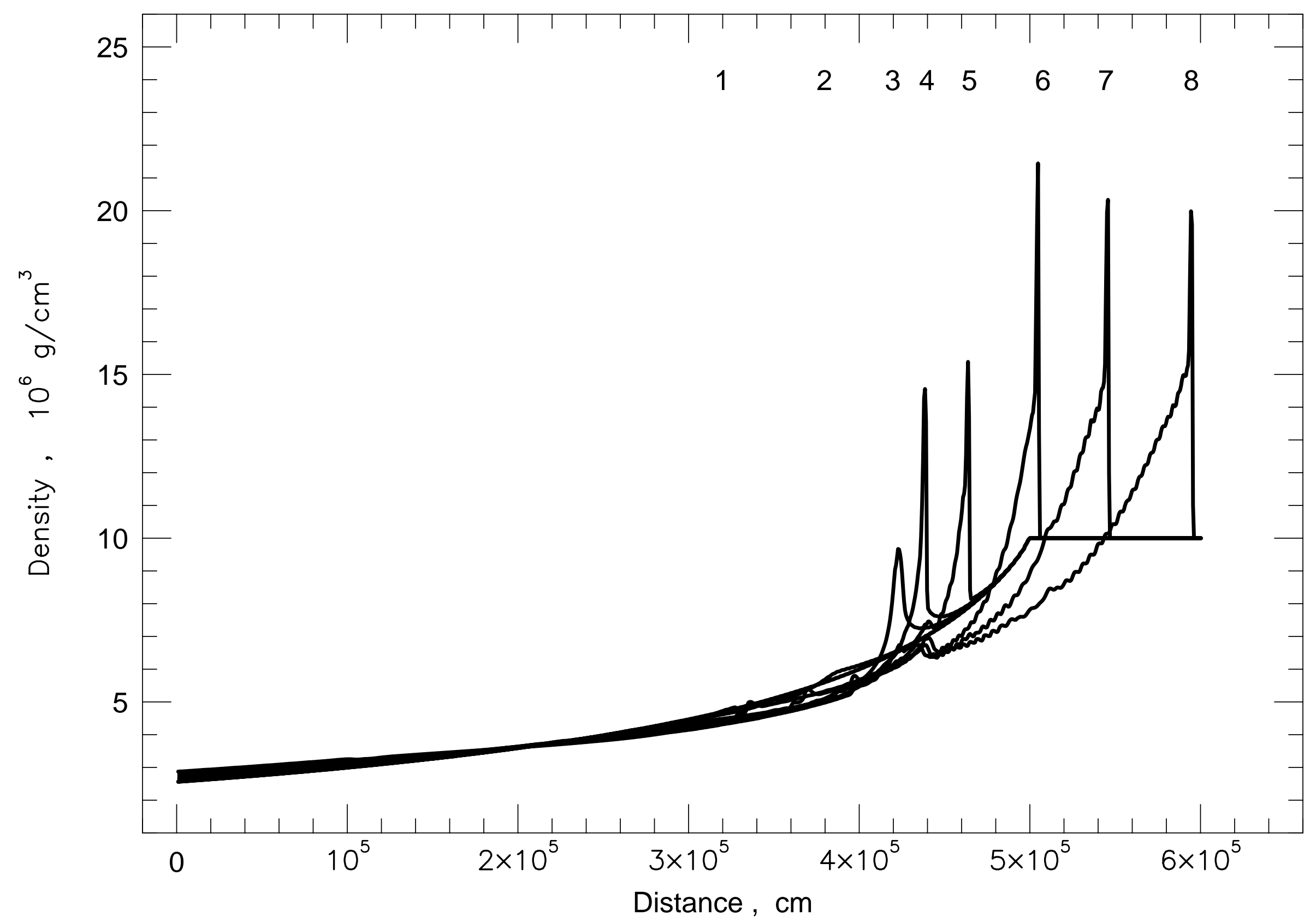




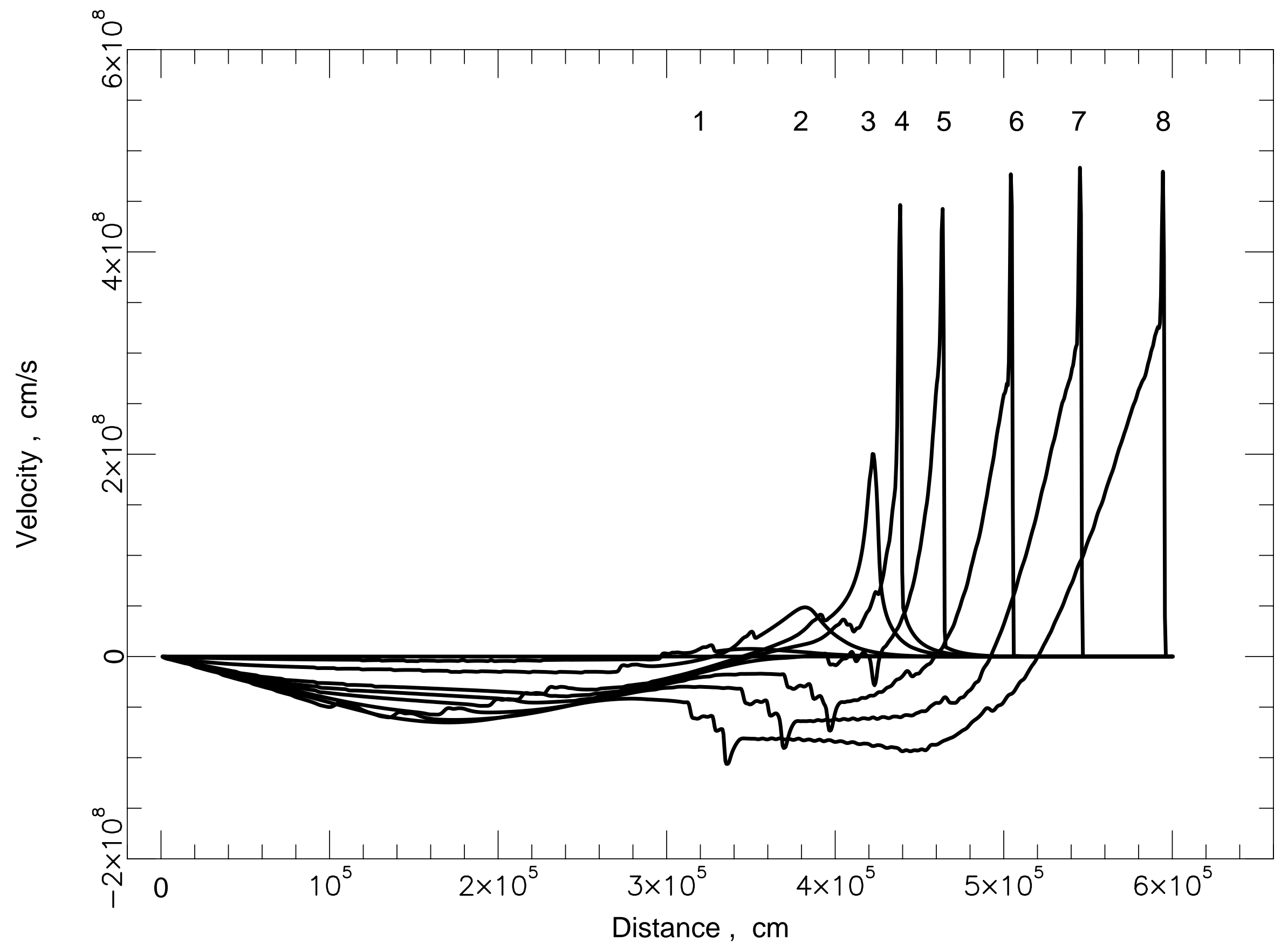




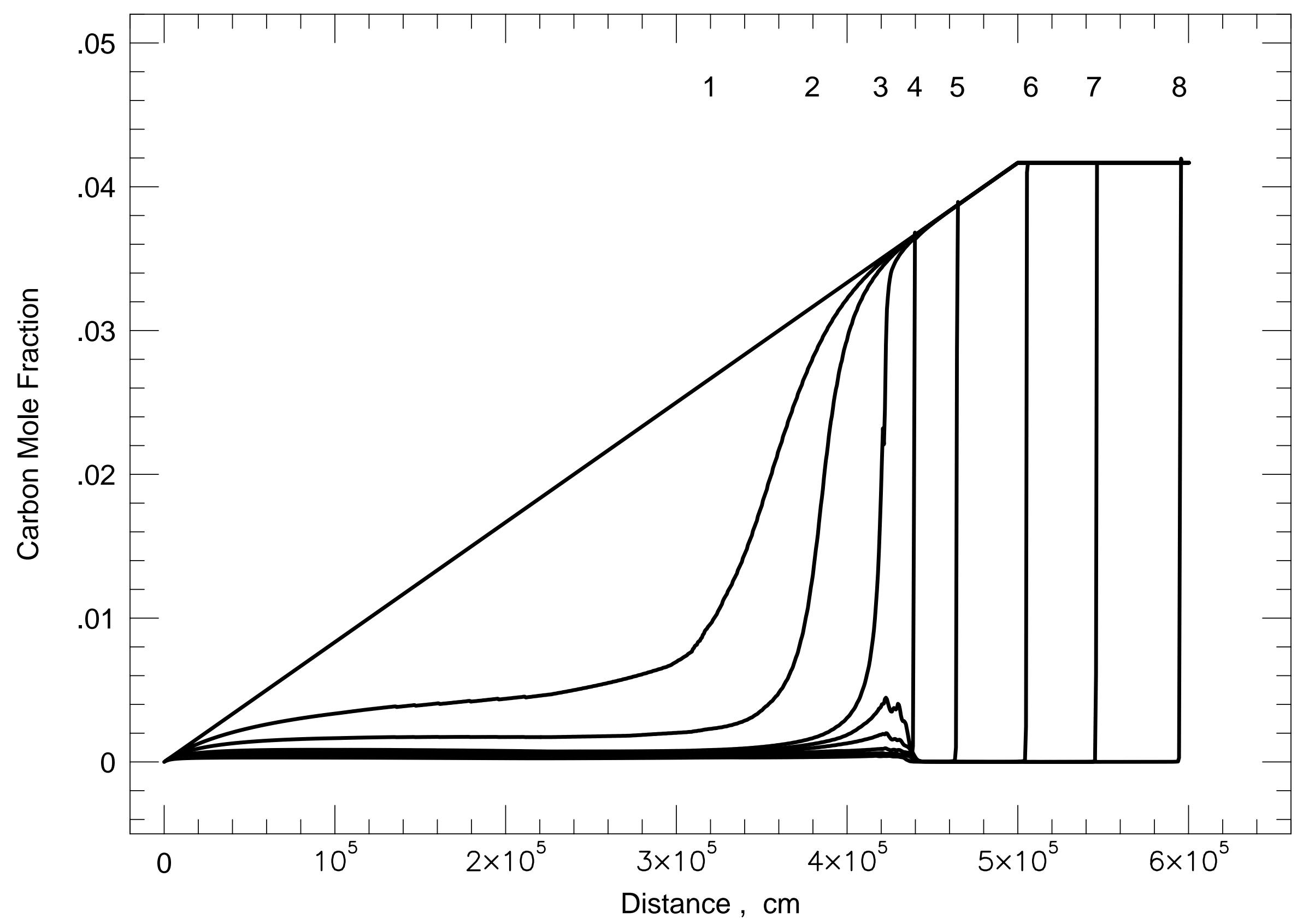




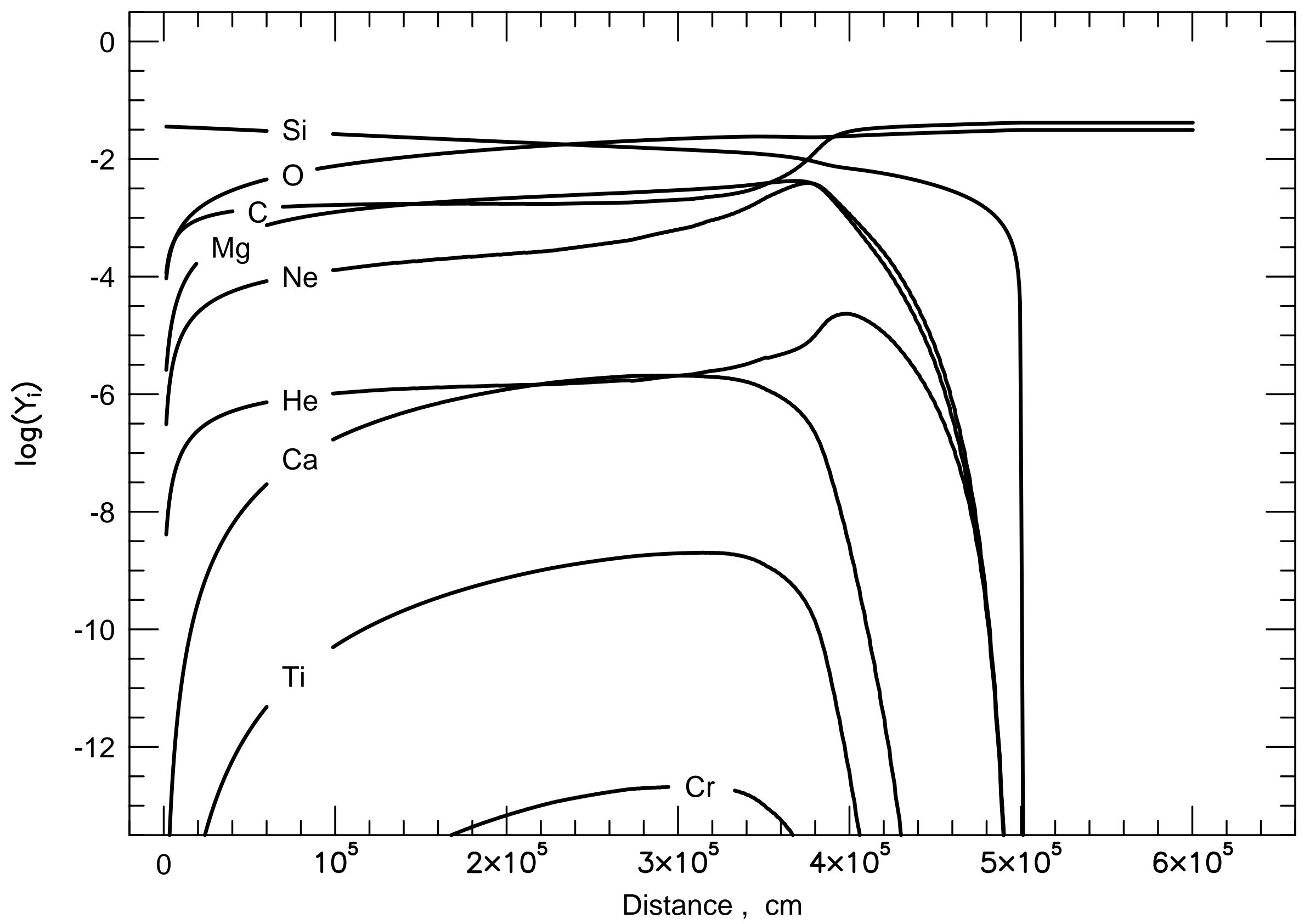




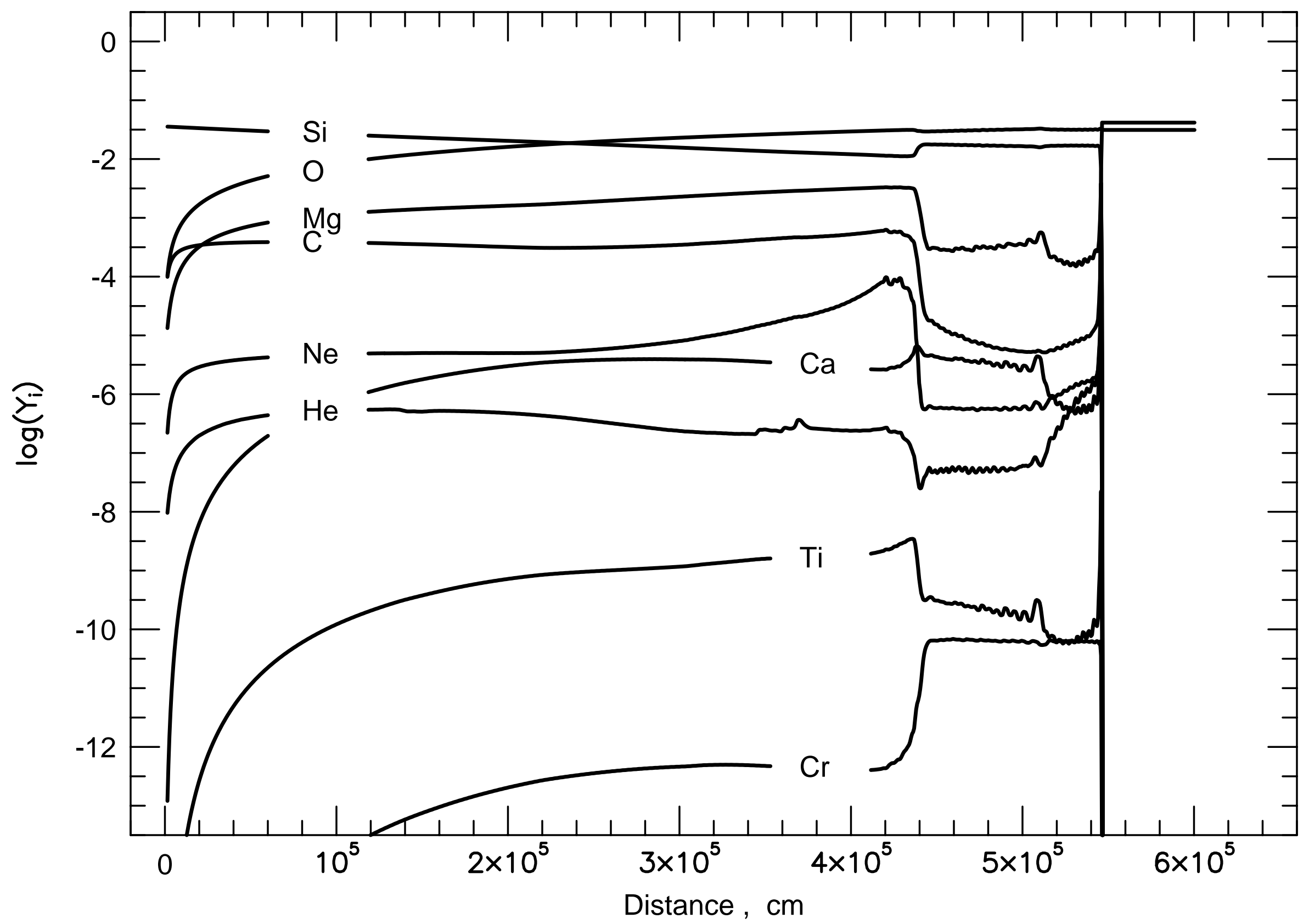




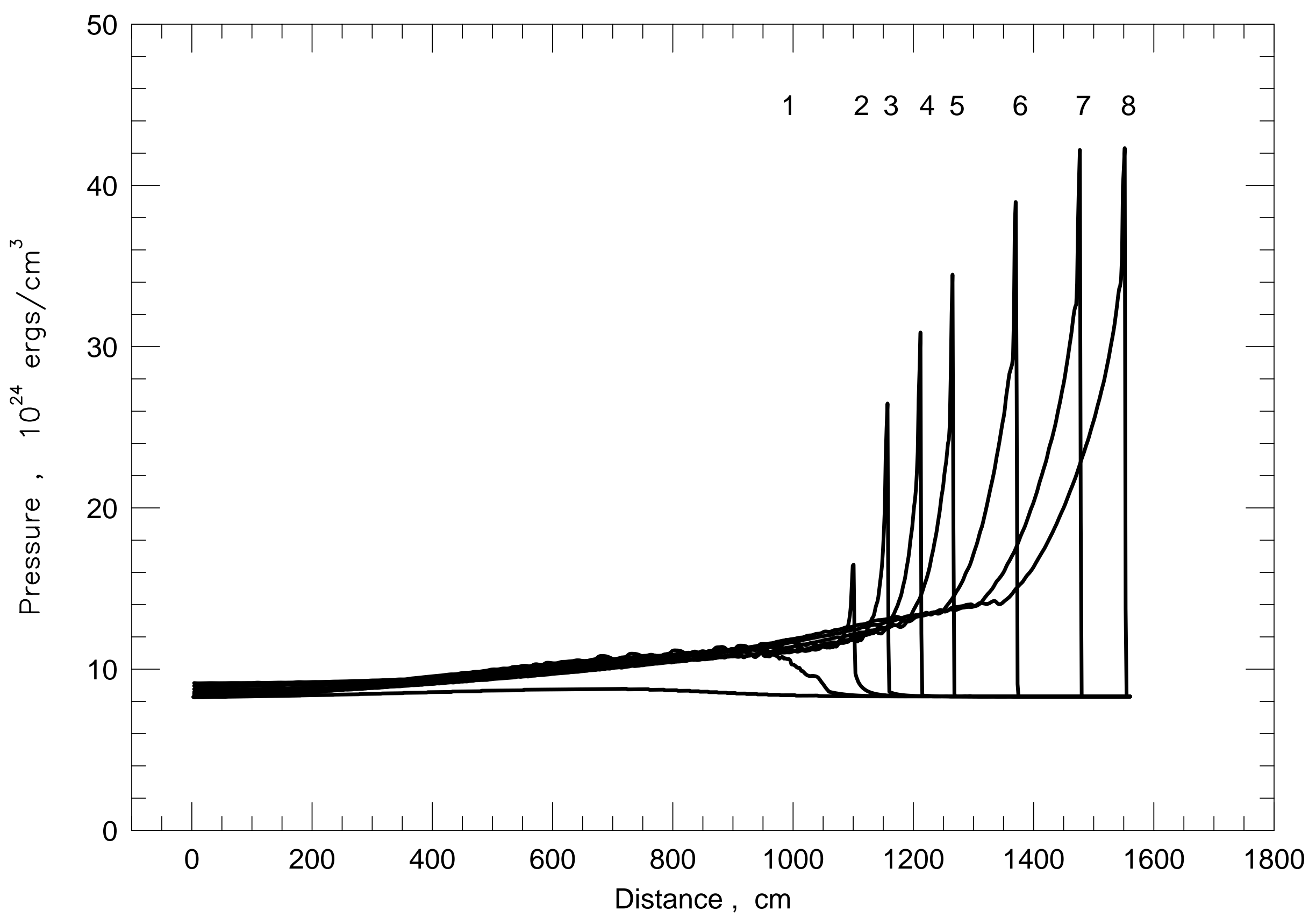




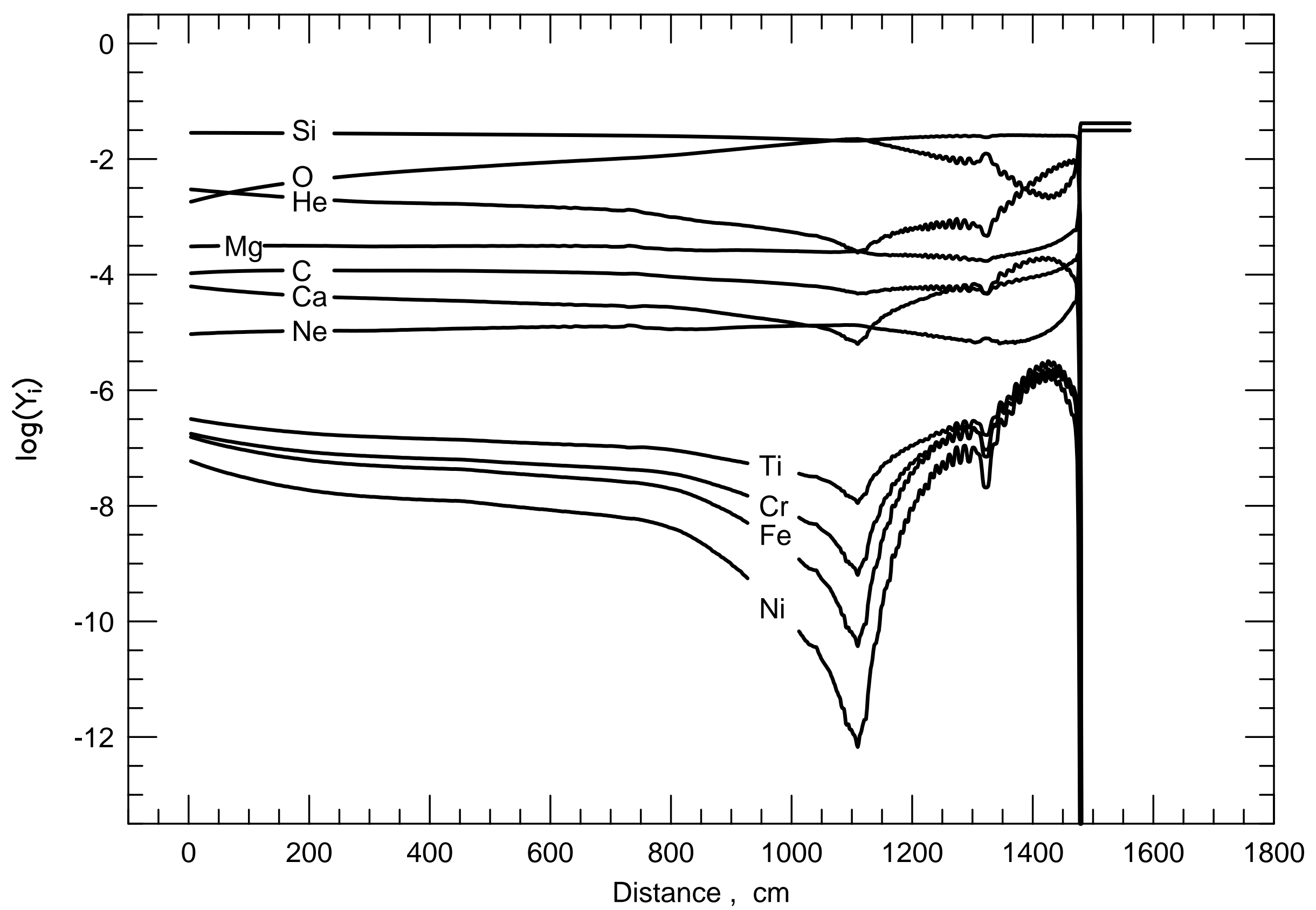




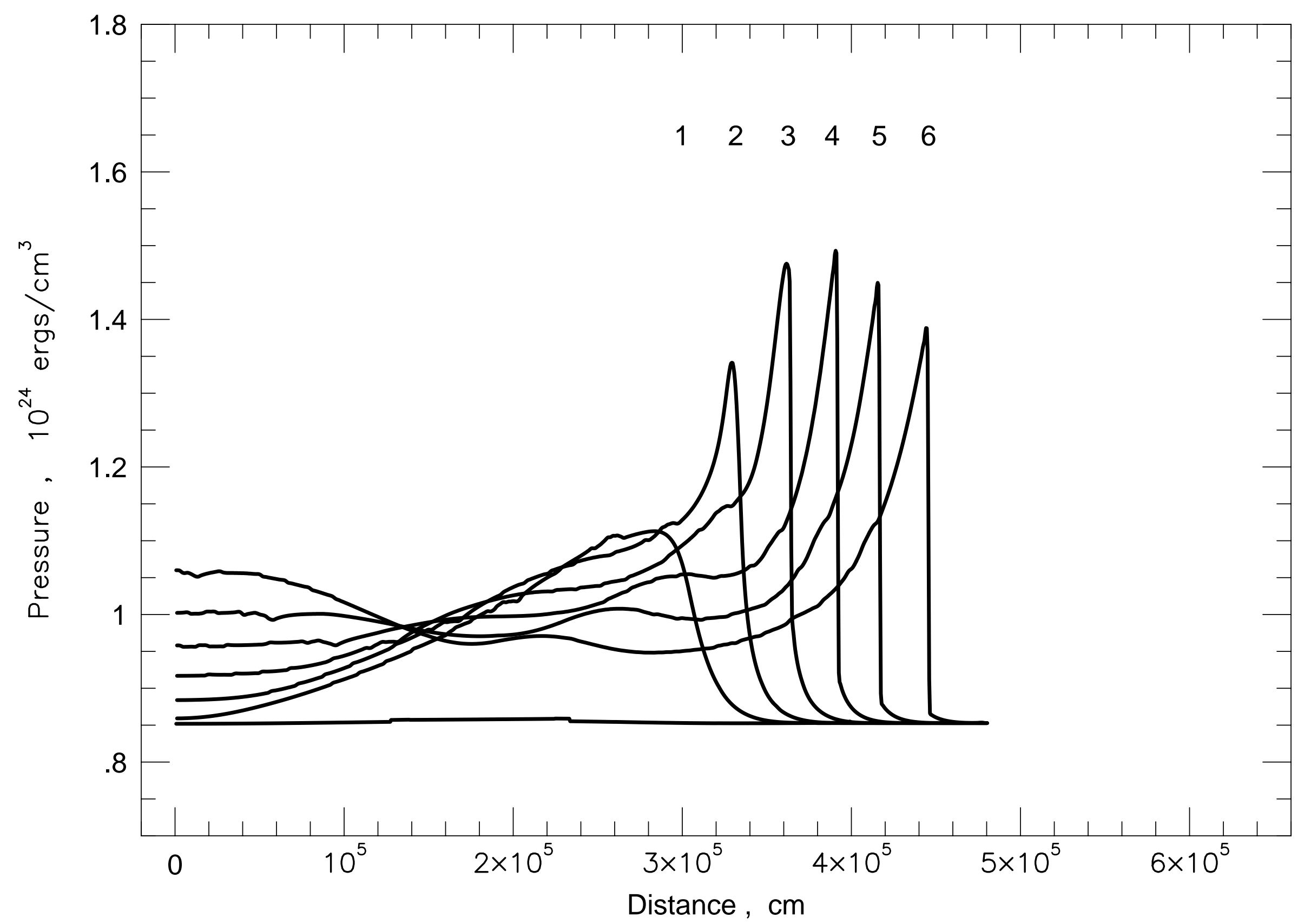




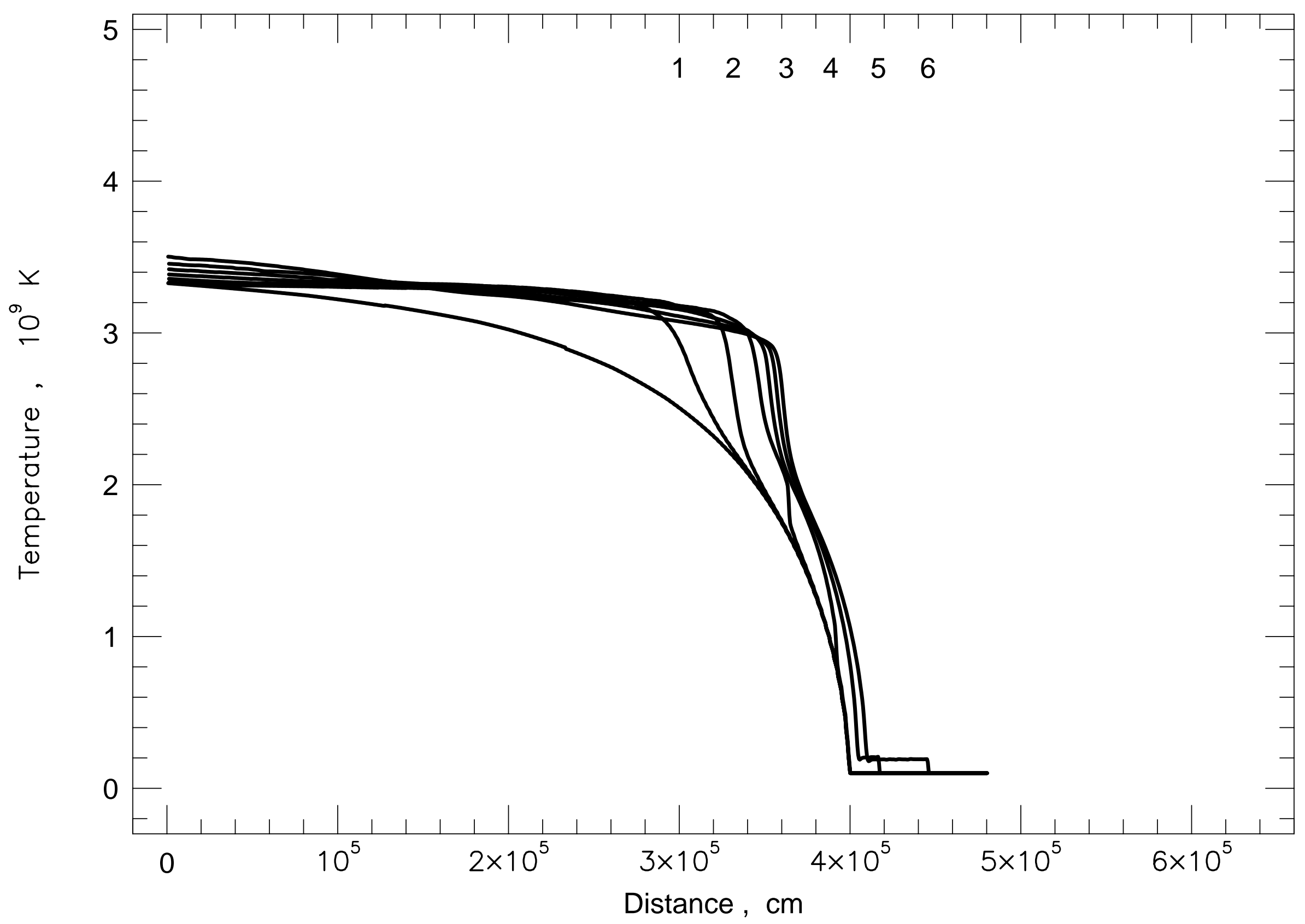




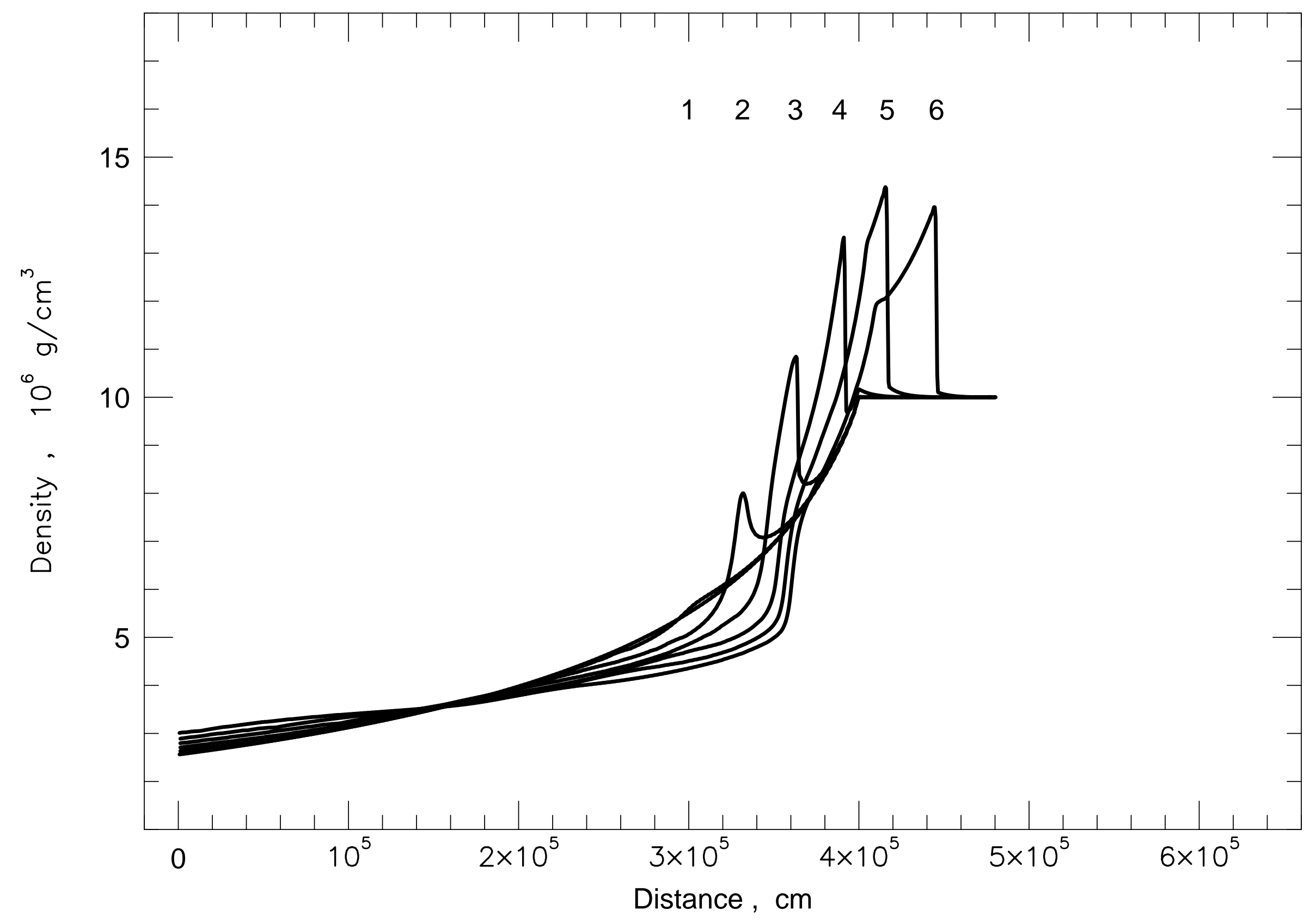




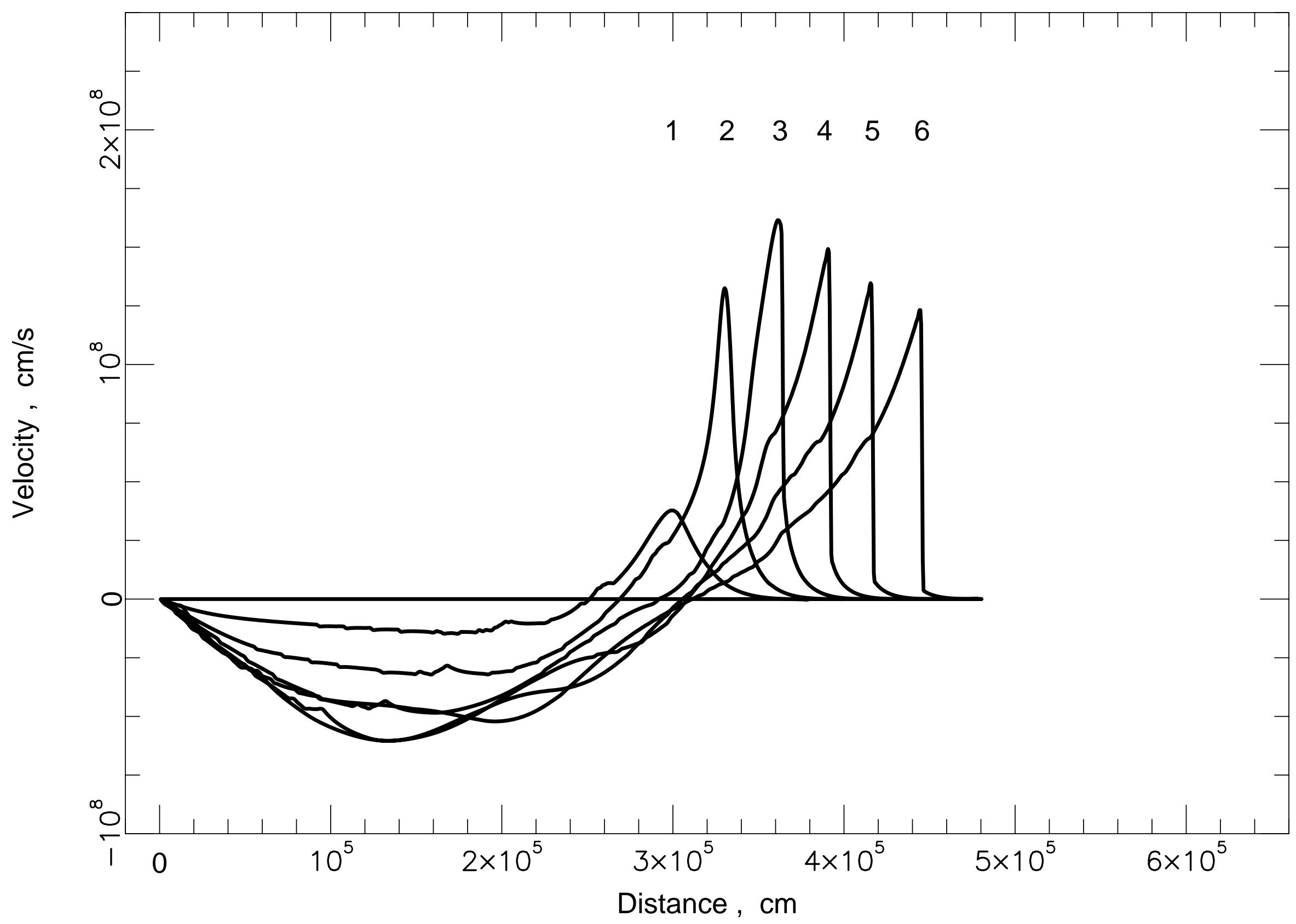




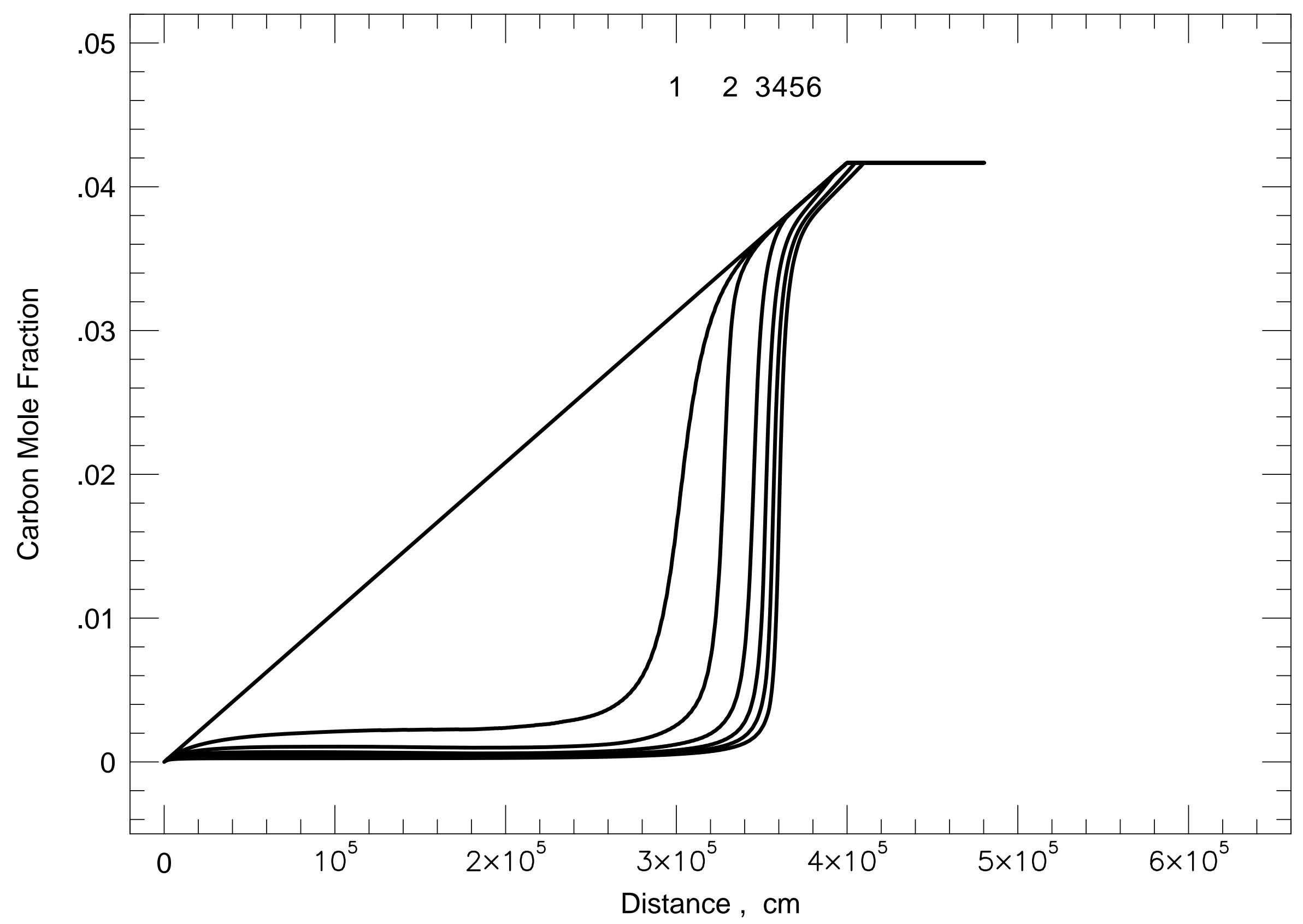




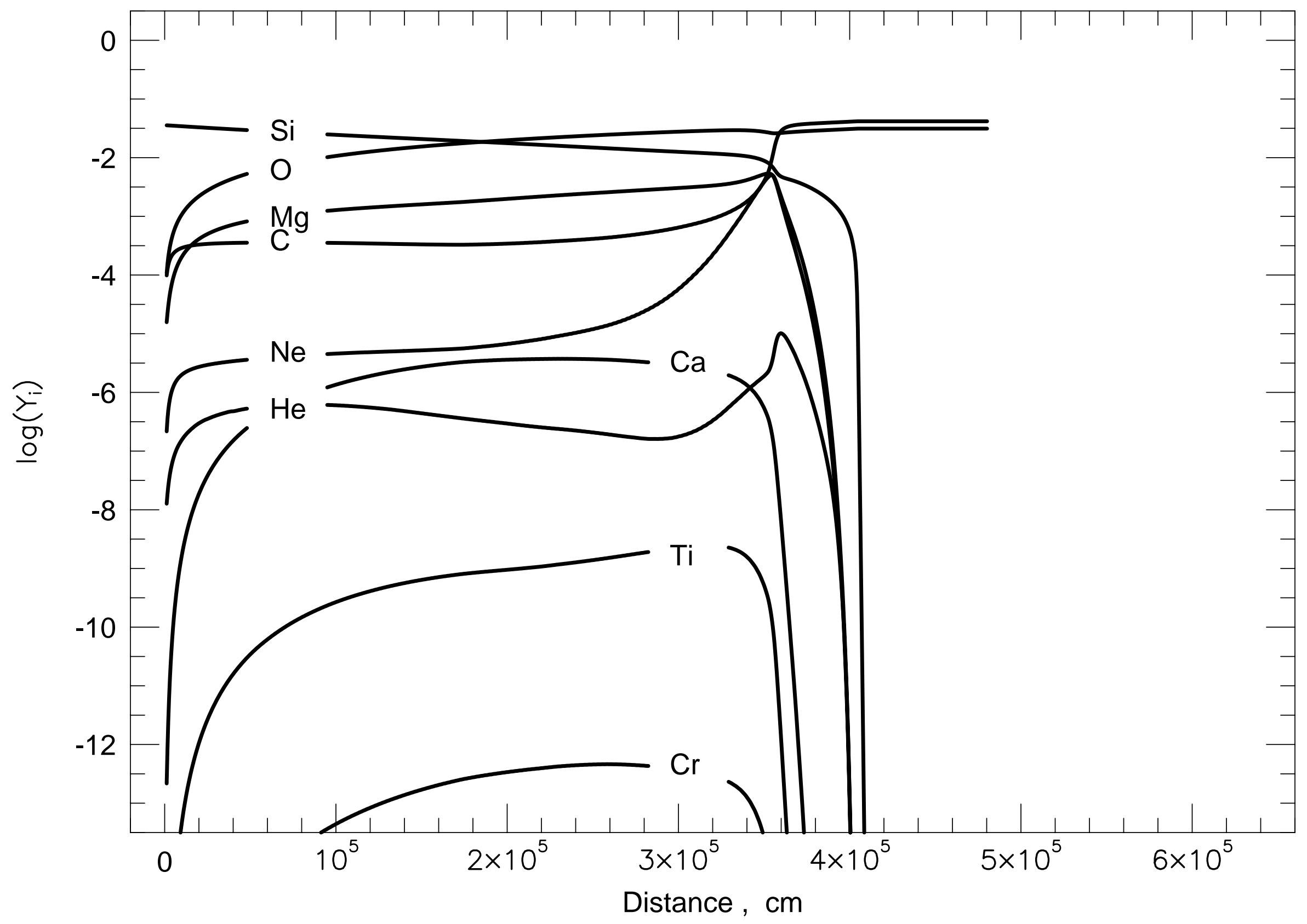




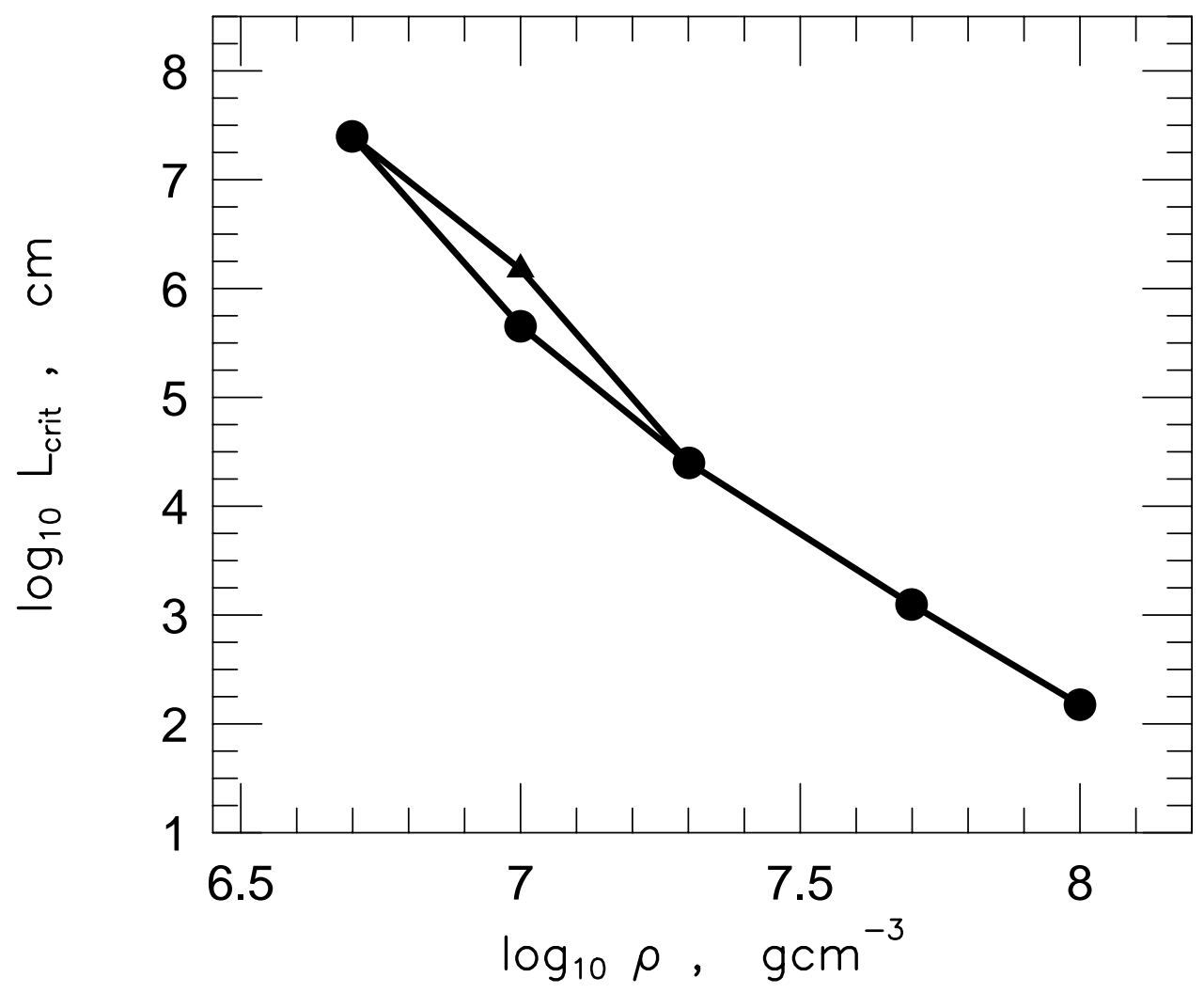




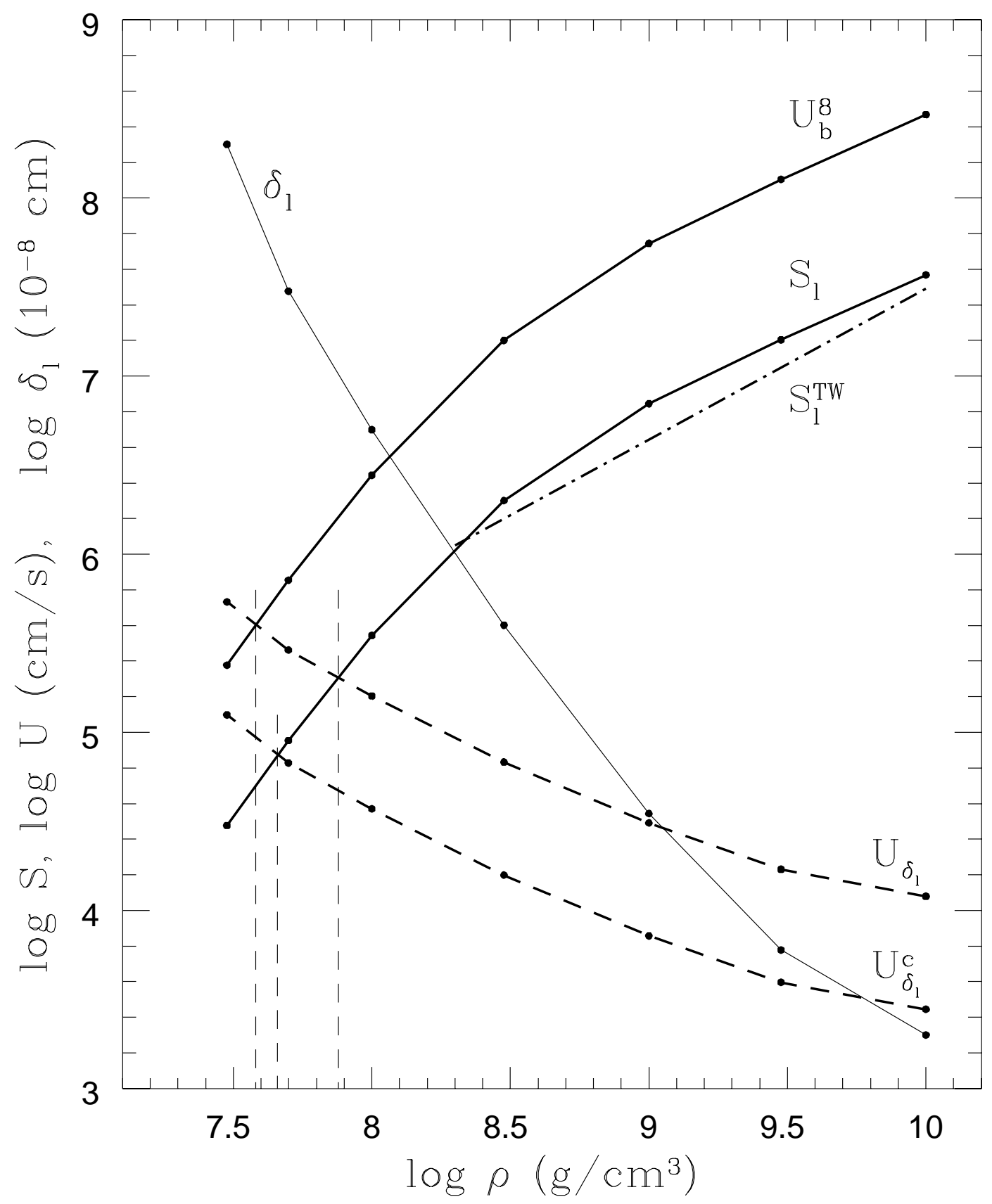

